\title{
Scalar neutrino dark matter in $\mathrm{U}(1)_{\times} \mathrm{SSM}$
}

\author{
Shu-Min Zhao, ${ }^{a, b}$ Tai-Fu Feng, ${ }^{a, b, c}$ Ming-Jie Zhang,,$^{a, b}$ Jin-Lei Yang, ${ }^{a, b}$ \\ Hai-Bin Zhang ${ }^{a, b}$ and Guo-Zhu Ning ${ }^{a, b}$ \\ ${ }^{a}$ Department of Physics, Hebei University, \\ Wusi road, Baoding, China \\ ${ }^{b}$ Key Laboratory of High-precision Computation and \\ Application of Quantum Field Theory of Hebei Province, Hebei University, \\ Wusi road, Baoding, China \\ ${ }^{c}$ Department of Physics, Chongqing University, \\ Shazheng street, Chongqing, China \\ E-mail: zhaosm@hbu.edu.cn, fengtf@hbu.edu.cn, 1070102415@qq.com, \\ JLYang JL@163 . com, hbzhang@hbu . edu . cn, ninggz@hbu . edu . cn
}

ABSTRACT: $\mathrm{U}(1)_{X} \mathrm{SSM}$ is the extension of the minimal supersymmetric standard model $(\mathrm{MSSM})$ and its local gauge group is $\mathrm{SU}(3)_{C} \times \mathrm{SU}(2)_{L} \times \mathrm{U}(1)_{Y} \times \mathrm{U}(1)_{X}$. To obtain this model, three singlet new Higgs superfields and right-handed neutrinos are added to MSSM. In the framework of $\mathrm{U}(1)_{X} \mathrm{SSM}$, we study the Higgs mass and take the lightest CP-even sneutrino as a cold dark matter candidate. For the lightest CP-even sneutrino, the relic density and the cross section for dark matter scattering off nucleon are both researched. In suitable parameter space of the model, the numerical results satisfy the constraints of the relic density and the cross section with the nucleon.

KeYwords: Extended Supersymmetry, Scattering Amplitudes

ARXIV EPRINT: 1905.11007 


\section{Contents}

1 Introduction 1

2 The $\mathrm{U}(1)_{X} \mathrm{SSM} \quad 2$

$\begin{array}{llr}3 & \text { Relic density } & 8\end{array}$

4 Direct detection $\quad 9$

5 Numerical results $\quad 10$

5.1 Higgs mass 11

$\begin{array}{lll}5.2 & \text { Relic density of sneutrino dark matter } & 12\end{array}$

$\begin{array}{ll}\text { 5.3 The cross section of the sneutrino scattering off nucleon } & 14\end{array}$

$\begin{array}{llr}6 & \text { Discussion and conclusion } & 14\end{array}$

$\begin{array}{ll}\text { A Mass matrix } & 16\end{array}$

\section{Introduction}

From the cosmological observations, astronomers are sure about the existence of dark matter in the universe, whose contribution is about five times that of visible matter [1,2]. Various luminous objects (stars, gas clouds globular clusters, or entire galaxies), moving faster than expectations $[3,4]$, are the earliest and the most compelling evidences for dark matter [5-8]. Dark matter must be electrically and color neutral, and can only take part in weak interactions. Dark matter is stable and has a long life-time $[9,10]$. At present, the mass and interaction properties of the dark matter are unknown.

Though the standard model (SM) successfully predicts the detection of the CP-even Higgs $(125.1 \mathrm{GeV})[11,12]$, it can not explain the relic density of dark matter in the universe. The relic density of light neutrinos with tiny mass is $\Omega_{\nu} h^{2} \leq 0.0062$ at $95 \%$ confidence level, that is much smaller than non-baryonic matter density $\Omega_{\nu} h^{2}=0.1186 \pm$ 0.0020 [13]. As a result, there must exist new physics beyond the SM. There are several dark matter candidates: axions, sterile neutrinos, primordial black holes and weakly interacting massive particles (WIMPs) $[9,14,15]$. WIMP, in particular, ranks among the most popular candidates for dark matter, whose detection is crucial for both distinguishing new physics models and understanding the nature of dark matter. The direct detection for dark matter is studying the recoil energy of nuclei caused by the elastic scattering of a WIMP off a nucleon. 
The neutralino in the minimal supersymmetric standard model (MSSM) has been extensively studied [16] as one of the favorite dark matter candidates. However, the lefthanded sneutrino meets severe troubles because the cross section for elastic scattering off nuclei exceeds the experimental limit by several orders with the exchange of vector boson $\mathrm{Z}$ [17]. Considering the neutrino oscillations, neutrino should possess tiny mass [18, 19]. Thus, to obtain light neutrino mass, one can add right-handed neutrino to the MSSM. The supersymmetric partners of the right-handed neutrinos will provide an alternative dark matter candidate [20-26]. There are also other works on sneutrino dark matter [22, 27-34]. At last but not least, it is worth mentioning that U(1) extensions of the MSSM considered in the works [35-39] have been of great interest lately.

In this work, we extend the MSSM to the $\mathrm{U}(1)_{X} \mathrm{SSM}$, whose local gauge group is $\mathrm{SU}(3)_{C} \times \mathrm{SU}(2)_{L} \times \mathrm{U}(1)_{Y} \times \mathrm{U}(1)_{X}$ [40-42]. In comparison with the MSSM, our model $\mathrm{U}(1)_{X} \mathrm{SSM}$ has more superfields: $\mathrm{U}(1)_{X}$ gauge field, righ-handed neutrinos, three $\mathrm{SU}(2)_{L}$ singlet Higgs superfields $\hat{\eta}, \hat{\bar{\eta}}, \hat{S}$ and their superpartners. The vacuum expectation value (VEV) of $\bar{\eta}$ produces masses of the right-handed neutrinos. The righ-handed neutrinos and left-handed neutrinos mix together through $Y_{\nu} \hat{\nu} \hat{l} \hat{H}_{u}$. Therefore, light neutrinos obtain tiny masses through the seesaw mechanism. The lightest sneutrino can be a new dark matter candidate different from the case in MSSM. Moreover, PAMELA [43] claims an excess in the electron/positron flux and no excess in the proton/antiproton flux [44]. Thus, the idea that dark matter carries lepton number is intriguing. The little hierarchy problem in MSSM is relieved in $\mathrm{U}(1)_{X} \mathrm{SSM}$ by the right-handed neutrinos, sneutrinos and additional Higgs singlets. U(1) $X$ SSM includes both terms $\mu \hat{H}_{u} \hat{H}_{d}$ and $\lambda_{H} \hat{S} \hat{H}_{u} \hat{H}_{d}$. When $\hat{S}$ develops a $\operatorname{VEV}\left(v_{S} / \sqrt{2}\right)$, an effective $\mu_{\text {eff }}$ is obtained as $\mu_{\text {eff }}=\mu+\lambda_{H} v_{S} / \sqrt{2}$, which can relieve the $\mu$ problem and even solve it. The spontaneously broken $\mathrm{U}(1)_{X}$ gauge symmetry can be used to avoid baryon number violating operators and keep proton stable. The interaction between three extra singlet Higgs superfields and two Higgs doublets is favorable to increase the mass of the lightest CP-even Higgs at the tree level. At the same time, the $\mathrm{U}(1)_{X} \mathrm{D}$-term gives another contribution. Considering both effects, large loop-induced contribution from stop sector is not necessary. Furthermore, the mass of the next light CP-even Higgs can reach the order of $\mathrm{TeV}$. The added parameters mitigate the constraints from experiments such as LHC.

We introduce the $\mathrm{U}(1)_{X} \mathrm{SSM}$ in detail in section II. Supposing the lightest CP-even sneutrino as a dark matter candidate, we study its relic density in section III. Section IV is devoted to research the direct detection for sneutrino elastic scattering off the nuclei. The numerical results for Higgs masses, relic density for dark matter and its direct detection are all presented in section V. Section VI is devoted to the discussions and conclusions.

\section{The $\mathrm{U}(1)_{X} \mathrm{SSM}$}

The gauge group of the $\mathrm{U}(1)_{X} \mathrm{SSM}$ is $\mathrm{SU}(3)_{C} \otimes \mathrm{SU}(2)_{L} \otimes \mathrm{U}(1)_{Y} \otimes \mathrm{U}(1)_{X}$. To obtain the $\mathrm{U}(1)_{X} \mathrm{SSM}$, new superfields are added to the MSSM, namely: three Higgs singlets $\hat{\eta}, \hat{\bar{\eta}}, \hat{S}$ and right-handed neutrinos $\hat{\nu}_{i}$. It can give light neutrino mass at the tree level through the seesaw mechanism. The neutral CP-even parts of $H_{u}, H_{d}, \eta, \bar{\eta}$ and $S$ mix together, 
forming $5 \times 5$ mass squared matrix. The loop corrections to the lightest CP-even Higgs are important and they are taken into account to get $125 \mathrm{GeV}$ Higgs mass $[45,46]$.

The superpotential for this model reads:

$$
\begin{aligned}
W= & l_{W} \hat{S}+\mu \hat{H}_{u} \hat{H}_{d}+M_{S} \hat{S} \hat{S}-Y_{d} \hat{d} \hat{q} \hat{H}_{d}-Y_{e} \hat{e} \hat{l} \hat{H}_{d}+\lambda_{H} \hat{S} \hat{H}_{u} \hat{H}_{d} \\
& +\lambda_{C} \hat{S} \hat{\eta} \hat{\bar{\eta}}+\frac{\kappa}{3} \hat{S} \hat{S} \hat{S}+Y_{u} \hat{u} \hat{q} \hat{H}_{u}+Y_{X} \hat{\nu} \hat{\bar{\eta}} \hat{\nu}+Y_{\nu} \hat{\nu} \hat{l} \hat{H}_{u} .
\end{aligned}
$$

There are two Higgs doublets and three Higgs singlets, whose explicit forms are shown in the follow,

$$
\begin{array}{rlrl}
H_{u} & =\left(\begin{array}{c}
H_{u}^{+} \\
\frac{1}{\sqrt{2}}\left(v_{u}+H_{u}^{0}+i P_{u}^{0}\right)
\end{array}\right), & H_{d}=\left(\begin{array}{c}
\frac{1}{\sqrt{2}}\left(v_{d}+H_{d}^{0}+i P_{d}^{0}\right) \\
H_{d}^{-}
\end{array}\right), \\
\eta & =\frac{1}{\sqrt{2}}\left(v_{\eta}+\phi_{\eta}^{0}+i P_{\eta}^{0}\right), & \bar{\eta}=\frac{1}{\sqrt{2}}\left(v_{\bar{\eta}}+\phi_{\bar{\eta}}^{0}+i P_{\bar{\eta}}^{0}\right), \\
S & =\frac{1}{\sqrt{2}}\left(v_{S}+\phi_{S}^{0}+i P_{S}^{0}\right) . & &
\end{array}
$$

$v_{u}, v_{d}, v_{\eta}, v_{\bar{\eta}}$ and $v_{S}$ are the corresponding VEVs of the Higgs superfields $H_{u}, H_{d}, \eta, \bar{\eta}$ and $S$. Here, we define $\tan \beta=v_{u} / v_{d}$ and $\tan \beta_{\eta}=v_{\bar{\eta}} / v_{\eta}$. The definition of $\tilde{\nu}_{L}$ and $\tilde{\nu}_{R}$ is

$$
\tilde{\nu}_{L}=\frac{1}{\sqrt{2}} \phi_{l}+\frac{i}{\sqrt{2}} \sigma_{l}, \quad \tilde{\nu}_{R}=\frac{1}{\sqrt{2}} \phi_{R}+\frac{i}{\sqrt{2}} \sigma_{R} .
$$

The soft SUSY breaking terms are

$$
\begin{aligned}
\mathcal{L}_{\mathrm{soft}}= & \mathcal{L}_{\mathrm{soft}}^{\mathrm{MSMS}}-B_{S} S^{2}-L_{S} S-\frac{T_{\kappa}}{3} S^{3}-T_{\lambda_{C}} S \eta \bar{\eta}+\epsilon_{i j} T_{\lambda_{H}} S H_{d}^{i} H_{u}^{j} \\
& -T_{X}^{I J} \bar{\eta} \tilde{\nu}_{R}^{* I} \tilde{\nu}_{R}^{* J}+\epsilon_{i j} T_{\nu}^{I J} H_{u}^{i} \tilde{\nu}_{R}^{I *} \tilde{l}_{j}^{J}-m_{\eta}^{2}|\eta|^{2}-m_{\bar{\eta}}^{2}|\bar{\eta}|^{2} \\
& -m_{S}^{2} S^{2}-\left(m_{\tilde{\nu}_{R}}^{2}\right)^{I J} \tilde{\nu}_{R}^{I *} \tilde{\nu}_{R}^{J}-\frac{1}{2}\left(M_{X} \lambda_{\tilde{X}}^{2}+2 M_{B B^{\prime}} \lambda_{\tilde{B}} \lambda_{\tilde{X}}\right)+\text { h.c. }
\end{aligned}
$$

The particle content and charge assignments for $\mathrm{U}(1)_{X} \mathrm{SSM}$ are shown in the table 1 . We use $Y^{Y}$ for representing the $\mathrm{U}(1)_{Y}$ charge and $Y^{X}$ for representing the $\mathrm{U}(1)_{X}$ charge. According to the textbook [47], the SM is anomaly free. The details regarding the absence of anomaly within the $\mathrm{U}(1)_{X} \mathrm{SSM}$ model can be summarized as follows:

1. The anomaly of three $\mathrm{SU}(2)_{L}$ gauge bosons vanishes as in the $\mathrm{SM}$ and the condition of three $\mathrm{SU}(3)_{C}$ gauge bosons is similar.

2. The anomalies containing one $\mathrm{SU}(3)_{C}$ boson or one $\mathrm{SU}(2)_{L}$ boson are proportional to $\operatorname{Tr}\left[t^{a}\right]=0$ or $\operatorname{Tr}\left[\tau^{a}\right]=0$.

3. The anomaly of one $\mathrm{U}(1)_{Y}$ or $\mathrm{U}(1)_{X}$ boson with two $\mathrm{SU}(3)_{C}$ bosons is proportional to the group theory factor $\operatorname{Tr}\left[t^{a} t^{b} Y^{Y}\right]=\frac{1}{2} \delta^{a b} \sum_{q} Y_{q}^{Y}$ or $\operatorname{Tr}\left[t^{a} t^{b} Y^{X}\right]=\frac{1}{2} \delta^{a b} \sum_{q} Y_{q}^{X}$.

4. The anomaly of one $\mathrm{U}(1)_{Y}$ or $\mathrm{U}(1)_{X}$ boson with two $\mathrm{SU}(2)_{L}$ bosons is proportional to $\operatorname{Tr}\left[\tau^{a} \tau^{b} Y^{Y}\right]=\frac{1}{2} \delta^{a b} \sum_{L} Y_{L}^{Y}$ or $\operatorname{Tr}\left[\tau^{a} \tau^{b} Y^{X}\right]=\frac{1}{2} \delta^{a b} \sum_{L} Y_{L}^{X}$. 


\begin{tabular}{|c|c|c|c|c|}
\hline Superfields & $\mathrm{SU}(3)_{C}$ & $\mathrm{SU}(2)_{L}$ & $\mathrm{U}(1)_{Y}$ & $\mathrm{U}(1)_{X}$ \\
\hline$\hat{Q}_{i}$ & 3 & 2 & $1 / 6$ & 0 \\
\hline$\hat{u}_{i}^{c}$ & $\overline{3}$ & 1 & $-2 / 3$ & $-1 / 2$ \\
\hline$\hat{d}_{i}^{c}$ & $\overline{3}$ & 1 & $1 / 3$ & $1 / 2$ \\
\hline$\hat{L}_{i}$ & 1 & 2 & $-1 / 2$ & 0 \\
\hline$\hat{e}_{i}^{c}$ & 1 & 1 & 1 & $1 / 2$ \\
\hline$\hat{\nu}_{i}$ & 1 & 1 & 0 & $-1 / 2$ \\
\hline$\hat{H}_{u}$ & 1 & 2 & $1 / 2$ & $1 / 2$ \\
\hline$\hat{H}_{d}$ & 1 & 2 & $-1 / 2$ & $-1 / 2$ \\
\hline$\hat{\eta}$ & 1 & 1 & 0 & -1 \\
\hline$\hat{\eta}$ & 1 & 1 & 0 & 1 \\
\hline$\hat{S}$ & 1 & 1 & 0 & 0 \\
\hline
\end{tabular}

Table 1. The superfields in $\mathrm{U}(1)_{X} \mathrm{SSM}$.

5. The anomalies of three $\mathrm{U}(1)$ gauge bosons are divided into four types

$$
\begin{array}{ll}
\operatorname{Tr}\left[Y^{Y} Y^{Y} Y^{Y}\right]=\sum_{n}\left(Y_{n}^{Y}\right)^{3}, & \operatorname{Tr}\left[Y^{X} Y^{X} Y^{X}\right]=\sum_{n}\left(Y_{n}^{X}\right)^{3}, \\
\operatorname{Tr}\left[Y^{X} Y^{Y} Y^{Y}\right]=\sum_{n} Y_{n}^{X}\left(Y_{n}^{Y}\right)^{2}, & \operatorname{Tr}\left[Y^{Y} Y^{X} Y^{X}\right]=\sum_{n} Y_{n}^{Y}\left(Y_{n}^{X}\right)^{2} .
\end{array}
$$

6. The gravitational anomaly with one $\mathrm{U}(1)$ gauge boson is proportional to $\operatorname{Tr}\left[Y^{Y}\right]=$ $\sum_{n} Y_{n}^{Y}$ or $\operatorname{Tr}\left[Y^{X}\right]=\sum_{n} Y_{n}^{X}$.

The anomalies that do not relate to $\mathrm{U}(1)_{X}$ are very similar as the SM condition and can be proved free easily. The anomalies including $\mathrm{U}(1)_{X}$ are also proved free, which are more complicated than those of SM. In the end, this model is anomaly free.

The presence of two Abelian groups $\mathrm{U}(1)_{Y}$ and $\mathrm{U}(1)_{X}$ in $\mathrm{U}(1)_{X} \mathrm{SSM}$ has a new effect absent in the MSSM with just one Abelian gauge group $\mathrm{U}(1)_{Y}$ : the gauge kinetic mixing. This effect can also be induced through RGEs, even if it is set to zero at $M_{\mathrm{GUT}}$.

The covariant derivatives of this model have the general form [39, 48-50]

$$
D_{\mu}=\partial_{\mu}-i(Y, X)\left(\begin{array}{cc}
g_{Y}, & g_{Y X}^{\prime} \\
g_{X Y}^{\prime}, & g_{X}^{\prime}
\end{array}\right)\left(\begin{array}{c}
A_{\mu}^{\prime Y} \\
A_{\mu}^{\prime X}
\end{array}\right) .
$$

Here, $A_{\mu}^{\prime Y}$ and $A_{\mu}^{\prime X}$ denote the gauge fields of $\mathrm{U}(1)_{Y}$ and $\mathrm{U}(1)_{X}$, while $Y$ and $X$ represent the hypercharge and $X$ charge respectively. We can perform a basis transformation, because the two Abelian gauge groups are unbroken. The following formula can be obtained with a correct matrix $R[39,49,50]$

$$
\left(\begin{array}{cc}
g_{Y}, & g_{Y X}^{\prime} \\
g_{X Y}^{\prime}, & g_{X}^{\prime}
\end{array}\right) R^{T}=\left(\begin{array}{cc}
g_{1}, & g_{Y X} \\
0, & g_{X}
\end{array}\right) .
$$


So the $\mathrm{U}(1)$ gauge fields are redefined as

$$
R\left(\begin{array}{c}
A_{\mu}^{Y} \\
A_{\mu}^{\prime X}
\end{array}\right)=\left(\begin{array}{c}
A_{\mu}^{Y} \\
A_{\mu}^{X}
\end{array}\right)
$$

The interesting thing is that the gauge bosons $A_{\mu}^{X}, A_{\mu}^{Y}$ and $V_{\mu}^{3}$ mix together at the tree level, and the mass matrix is shown in the basis $\left(A_{\mu}^{Y}, V_{\mu}^{3}, A_{\mu}^{X}\right)$

$$
\left(\begin{array}{ccc}
\frac{1}{8} g_{1}^{2} v^{2} & -\frac{1}{8} g_{1} g_{2} v^{2} & \frac{1}{8} g_{1} g_{Y X} v^{2} \\
-\frac{1}{8} g_{1} g_{2} v^{2} & \frac{1}{8} g_{2}^{2} v^{2} & -\frac{1}{8} g_{2} g_{Y X} v^{2} \\
\frac{1}{8} g_{1} g_{Y X} v^{2} & -\frac{1}{8} g_{2} g_{Y X} v^{2} & \frac{1}{8} g_{Y X}^{2} v^{2}+\frac{1}{8} g_{X}^{2} \xi^{2}
\end{array}\right),
$$

with $v^{2}=v_{u}^{2}+v_{d}^{2}$ and $\xi^{2}=v_{\eta}^{2}+v_{\bar{\eta}}^{2}$. To diagonalize the mass matrix in eq. (2.9), an unitary matrix including two mixing angles $\theta_{W}$ and $\theta_{W}^{\prime}$ is used here

$$
\left(\begin{array}{c}
\gamma_{\mu} \\
Z_{\mu} \\
Z_{\mu}^{\prime}
\end{array}\right)=\left(\begin{array}{ccc}
\cos \theta_{W} & \sin \theta_{W} & 0 \\
-\sin \theta_{W} \cos \theta_{W}^{\prime} & \cos \theta_{W} \cos \theta_{W}^{\prime} & \sin \theta_{W}^{\prime} \\
\sin \theta_{W} \sin \theta_{W}^{\prime} & -\cos \theta_{W}^{\prime} \sin \theta_{W}^{\prime} & \cos \theta_{W}^{\prime}
\end{array}\right)\left(\begin{array}{c}
A_{\mu}^{Y} \\
V_{\mu}^{3} \\
A_{\mu}^{X}
\end{array}\right) .
$$

We deduce $\sin ^{2} \theta_{W}^{\prime}$ as

$$
\sin ^{2} \theta_{W}^{\prime}=\frac{1}{2}-\frac{\left(g_{Y X}^{2}-g_{1}^{2}-g_{2}^{2}\right) v^{2}+4 g_{X}^{2} \xi^{2}}{2 \sqrt{\left(g_{Y X}^{2}+g_{1}^{2}+g_{2}^{2}\right)^{2} v^{4}+8 g_{X}^{2}\left(g_{Y X}^{2}-g_{1}^{2}-g_{2}^{2}\right) v^{2} \xi^{2}+16 g_{X}^{4} \xi^{4}}} .
$$

The new mixing angle $\theta_{W}^{\prime}$ appears in the couplings involving $Z$ and $Z^{\prime}$. The exact eigenvalues of eq. (2.9) are calculated [39, 49, 50]

$$
\begin{aligned}
m_{\gamma}^{2}= & 0, \\
m_{Z, Z^{\prime}}^{2}= & \frac{1}{8}\left(\left(g_{1}^{2}+g_{2}^{2}+g_{Y X}^{2}\right) v^{2}+4 g_{X}^{2} \xi^{2}\right. \\
& \left.\mp \sqrt{\left(g_{1}^{2}+g_{2}^{2}+g_{Y X}^{2}\right)^{2} v^{4}+8\left(g_{Y X}^{2}-g_{1}^{2}-g_{2}^{2}\right) g_{X}^{2} v^{2} \xi^{2}+16 g_{X}^{4} \xi^{4}}\right) .
\end{aligned}
$$

The Higgs potential is deduced here

$$
\begin{aligned}
V= & \frac{1}{2} g_{X}\left(g_{X}+g_{Y X}\right)\left(\left|H_{d}^{0}\right|^{2}-\left|H_{u}^{0}\right|^{2}\right)\left(|\eta|^{2}-|\bar{\eta}|^{2}\right)+\left|\lambda_{H}\right|^{2}\left|H_{u}^{0} H_{d}^{0}\right|^{2}+m_{S}^{2}|S|^{2} \\
& +\frac{1}{8}\left(g_{1}^{2}+g_{2}^{2}+\left(g_{X}+g_{Y X}\right)^{2}\right)\left(\left|H_{d}^{0}\right|^{2}-\left|H_{u}^{0}\right|^{2}\right)^{2}+\frac{1}{2} g_{X}^{2}\left(|\eta|^{2}-|\bar{\eta}|^{2}\right)^{2}+\lambda_{C}^{2}|\eta \bar{\eta}|^{2} \\
& +\left(|\mu|^{2}+\left|\lambda_{H}\right|^{2}|S|^{2}+2 \operatorname{Re}\left[\mu^{*} \lambda_{H} S\right]\right)\left(\left|H_{d}^{0}\right|^{2}+\left|H_{u}^{0}\right|^{2}\right)+\left|\lambda_{C}\right|^{2}|S|^{2}\left(|\eta|^{2}+|\bar{\eta}|^{2}\right) \\
& +2 \operatorname{Re}\left[l_{W}^{*}\left(2 M_{S} S+\lambda_{C} \eta \bar{\eta}-\lambda_{H} H_{u}^{0} H_{d}^{0}+\kappa S^{2}\right)\right]+4\left|M_{S}\right|^{2}|S|^{2}+2 \operatorname{Re}\left[\lambda_{C}^{*} \kappa \eta^{*} \bar{\eta}^{*} S^{2}\right] \\
& +|\kappa|^{2}|S|^{4}+4 \operatorname{Re}\left[M_{S}^{*} S^{*}\left(\lambda_{C} \eta \bar{\eta}-\lambda_{H} H_{u}^{0} H_{d}^{0}+\kappa S^{2}\right)\right]-2 \operatorname{Re}\left[\lambda_{C}^{*} \lambda_{H} \eta^{*} \bar{\eta}^{*} H_{u}^{0} H_{d}^{0}\right]+\left|l_{W}\right|^{2}
\end{aligned}
$$




$$
\begin{aligned}
& -2 \operatorname{Re}\left[B_{\mu} H_{d}^{0} H_{u}^{0}\right]+2 \operatorname{Re}\left[L_{S} S\right]+\frac{2}{3} \operatorname{Re}\left[T_{k} S^{3}\right]+2 \operatorname{Re}\left[T_{\lambda_{C}} \eta \bar{\eta} S\right]-2 \operatorname{Re}\left[T_{\lambda_{H}} H_{d}^{0} H_{u}^{0} S\right] \\
& -2 \operatorname{Re}\left[\lambda_{H} \kappa^{*} H_{u}^{0} H_{d}^{0}\left(S^{2}\right)^{*}\right]+m_{\eta}^{2}|\eta|^{2}+m_{\bar{\eta}}^{2}|\bar{\eta}|^{2}+m_{H_{u}^{0}}^{2}\left|H_{u}^{0}\right|^{2}+m_{H_{d}^{0}}^{2}\left|H_{d}^{0}\right|^{2}+2 \operatorname{Re}\left[B_{S} S^{2}\right] .
\end{aligned}
$$

To simplify the following discussion, we suppose that the parameters $\left(\mu, \lambda_{H}, \lambda_{C}, l_{W}\right.$, $\left.M_{S}, B_{\mu}, L_{S}, T_{\kappa}, T_{\lambda_{C}}, T_{\lambda_{H}}, \kappa, B_{S}\right)$ in eq. (2.13) are real parameters. The VEVs of the Higgs satisfy the following equations

$$
\begin{gathered}
\frac{1}{8}\left(g_{1}^{2}+g_{2}^{2}+\left(g_{X}+g_{Y X}\right)^{2}\right)\left(v_{d}^{2}-v_{u}^{2}\right) v_{d}+\frac{1}{4} g_{X}\left(g_{X}+g_{Y X}\right) v_{d}\left(v_{\eta}^{2}-v_{\bar{\eta}}^{2}\right) \\
+\left(\mu^{2}+\frac{1}{2} \lambda_{H}^{2} v_{S}^{2}+\sqrt{2} \mu \lambda_{H} v_{S}\right) v_{d}-l_{W} \lambda_{H} v_{u}+\frac{1}{2} \lambda_{H}^{2} v_{u}^{2} v_{d}-\sqrt{2} M_{S} \lambda_{H} v_{S} v_{u} \\
-\frac{1}{2} \lambda_{H} \lambda_{C} v_{\eta} v_{\bar{\eta}} v_{u}-\frac{1}{2} \lambda_{H} \kappa v_{u} v_{S}^{2}+m_{H_{d}}^{2} v_{d}-B_{\mu} v_{u}-\frac{T_{\lambda_{H}}}{\sqrt{2}} v_{u} v_{S}=0 \\
\frac{1}{8}\left(g_{1}^{2}+g_{2}^{2}+\left(g_{X}+g_{Y X}\right)^{2}\right)\left(v_{u}^{2}-v_{d}^{2}\right) v_{u}+\frac{1}{4} g_{X}\left(g_{X}+g_{Y X}\right) v_{u}\left(v_{\bar{\eta}}^{2}-v_{\eta}^{2}\right) \\
+\left(\mu^{2}+\frac{1}{2} \lambda_{H}^{2} v_{S}^{2}+\sqrt{2} \mu \lambda_{H} v_{S}\right) v_{u}-l_{W} \lambda_{H} v_{d}+\frac{1}{2} \lambda_{H}^{2} v_{u} v_{d}^{2}-\sqrt{2} M_{S} \lambda_{H} v_{S} v_{d} \\
-\frac{1}{2} \lambda_{H} \lambda_{C} v_{\eta} v_{\bar{\eta}} v_{d}-\frac{1}{2} \lambda_{H} \kappa v_{d} v_{S}^{2}+m_{H_{u}}^{2} v_{u}-B_{\mu} v_{d}-\frac{T_{\lambda_{H}}}{\sqrt{2}} v_{d} v_{S}=0 \\
\frac{1}{2} g_{X}^{2}\left(v_{\eta}^{2}-v_{\bar{\eta}}^{2}\right) v_{\eta}-\frac{1}{4} g_{X}\left(g_{X}+g_{Y X}\right) v_{\eta}\left(v_{u}^{2}-v_{d}^{2}\right)+\frac{1}{2} \lambda_{C}^{2} v_{\eta}\left(v_{S}^{2}+v_{\bar{\eta}}^{2}\right)+l_{W} \lambda_{C} v_{\bar{\eta}} \\
+\sqrt{2} M_{S} \lambda_{C} v_{S} v_{\bar{\eta}}-\frac{1}{2} \lambda_{H} \lambda_{C} v_{\bar{\eta}} v_{u} v_{d}+\frac{1}{2} \lambda_{C} \kappa v_{\bar{\eta}} v_{S}^{2}+m_{\eta}^{2} v_{\eta}+\frac{T_{\lambda_{H}}}{\sqrt{2}} v_{\bar{\eta}} v_{S}=0 \\
\frac{1}{2} g_{X}^{2}\left(v_{\bar{\eta}}^{2}-v_{\eta}^{2}\right) v_{\bar{\eta}}+\frac{1}{4} g_{X}\left(g_{X}+g_{Y X}\right) v_{\bar{\eta}}\left(v_{u}^{2}-v_{d}^{2}\right)+\frac{1}{2} \lambda_{C}^{2} v_{\bar{\eta}}\left(v_{S}^{2}+v_{\eta}^{2}\right)+l_{W} \lambda_{C} v_{\eta} \\
+\sqrt{2} M_{S} \lambda_{C} v_{S} v_{\eta}-\frac{1}{2} \lambda_{H} \lambda_{C} v_{\eta} v_{u} v_{d}+\frac{1}{2} \lambda_{C} \kappa v_{\eta} v_{S}^{2}+m_{\bar{\eta}}^{2} v_{\bar{\eta}}+\frac{T_{\lambda_{H}}}{\sqrt{2}} v_{\eta} v_{S}=0 \\
+\lambda_{C} \kappa v_{\eta} v_{\bar{\eta}} v_{S}-\lambda_{H} \kappa v_{u} v_{d} v_{S}+\frac{1}{\sqrt{2}}\left(T_{k} v_{S}^{2}+T_{\lambda_{C}} v_{\eta} v_{\bar{\eta}}-T_{\lambda_{H}} v_{u} v_{d}\right)=0 \\
\left(\lambda_{H}^{2} v_{S}+\sqrt{2} \mu \lambda_{H}\right) \frac{1}{2} v^{2}+\frac{1}{2} \lambda_{C}^{2} v_{S} \xi^{2}+4 M_{S}^{2} v_{S}+\kappa^{2} v_{S}^{3}+2 B v_{S}+\sqrt{2} L_{S} \\
+2 l_{W}\left(\sqrt{2} M_{S}+\kappa v_{S}\right)+\sqrt{2} M_{S}\left(\lambda_{C} v_{\eta} v_{\bar{\eta}}-\lambda_{H} v_{u} v_{d}+3 \kappa v_{S}^{2}\right)+m_{S}^{2} v_{S}
\end{gathered}
$$

The mass squared matrix for CP-odd Higgs in the basis $\left(\sigma_{d}, \sigma_{u}, \sigma_{\eta}, \sigma_{\bar{\eta}}, \sigma_{s}\right)$ is diagonalized by $Z^{A}$. The neutral CP-even Higgs $\phi_{d}, \phi_{u}, \phi_{\eta}, \phi_{\bar{\eta}}$ and $\phi_{S}$ mix together at the tree level and they form $5 \times 5$ mass squared matrix which is diagonalized by $Z^{H}$. Their concrete forms are collected in the appendix. As discussed in the MSSM, the loop corrections to the lightest CP-even Higgs mass are known to be large. Therefore, we include the leading-log radiative corrections from stop and top particles [45, 46]. The mass of the lightest Higgs boson can be written as

$$
m_{h}=\sqrt{\left(m_{h_{1}}^{0}\right)^{2}+\Delta m_{h}^{2}}
$$


with $m_{h_{1}}^{0}$ representing the lightest tree-level Higgs boson mass. The concrete form of $\Delta m_{h}^{2}$ is

$$
\begin{aligned}
\Delta m_{h}^{2} & =\frac{3 m_{t}^{4}}{2 \pi v^{2}}\left[\left(\tilde{t}+\frac{1}{2}+\tilde{X}_{t}\right)+\frac{1}{16 \pi^{2}}\left(\frac{3 m_{t}^{2}}{2 v^{2}}-32 \pi \alpha_{3}\right)\left(\tilde{t}^{2}+\tilde{X}_{t} \tilde{t}\right)\right], \\
\tilde{t} & =\log \frac{M_{\tilde{\tilde{T}}}^{2}}{m_{t}^{2}}, \quad \tilde{X}_{t}=\frac{2 \tilde{A}_{t}^{2}}{M_{\tilde{T}}^{2}}\left(1-\frac{\tilde{A}_{t}^{2}}{12 M_{\tilde{T}}^{2}}\right) .
\end{aligned}
$$

$\alpha_{3}$ is the strong coupling constant. $M_{\tilde{T}}=\sqrt{m_{\tilde{t}_{1}} m_{\tilde{t}_{2}}}$ and $m_{\tilde{t}_{1,2}}$ are the stop masses. $\tilde{A}_{t}=$ $A_{t}-\mu \cot \beta$ and $A_{t}$ is the trilinear Higgs stop coupling.

The neutrino mass matrix is deduced in the base $\left(\nu_{L}, \bar{\nu}_{R}\right)$

$$
M_{\nu}=\left(\begin{array}{cc}
0 & \frac{v_{u}}{\sqrt{2}}\left(Y_{\nu}^{T}\right)^{I J} \\
\frac{v_{u}}{\sqrt{2}}\left(Y_{\nu}\right)^{I J} & \sqrt{2} v_{\bar{\eta}}\left(Y_{X}\right)^{I J}
\end{array}\right),
$$

and it is diagonalized by the matrix $Z_{\nu}$ through the formula

$$
Z_{\nu} M_{\nu} Z_{\nu}^{T}=\operatorname{diag}\left(M_{\nu}\right)
$$

The mass matrix for CP-even sneutrino $\left(\phi_{l}, \phi_{r}\right)$ reads

$$
\begin{aligned}
M_{\tilde{\nu} R}^{2}= & \left(\begin{array}{cc}
m_{\phi_{l} \phi_{l}} & m_{\phi_{r} \phi_{l}}^{T} \\
m_{\phi_{l} \phi_{r}} & m_{\phi_{r} \phi_{r}}
\end{array}\right) \\
m_{\phi_{l} \phi_{l}}= & \frac{1}{8}\left(\left(g_{1}^{2}+g_{Y X}^{2}+g_{2}^{2}+g_{Y X} g_{X}\right)\left(v_{d}^{2}-v_{u}^{2}\right)+g_{Y X} g_{X}\left(2 v_{\eta}^{2}-2 v_{\bar{\eta}}^{2}\right)\right) \\
& +\frac{1}{2} v_{u}^{2} Y_{\nu}^{T} Y_{\nu}+m_{\tilde{L}}^{2}, \\
m_{\phi_{l} \phi_{r}}= & \frac{1}{\sqrt{2}} v_{u} T_{\nu}+v_{u} v_{\bar{\eta}} Y_{X} Y_{\nu}-\frac{1}{2} v_{d}\left(\lambda_{H} v_{S}+\sqrt{2} \mu\right) Y_{\nu}, \\
m_{\phi_{r} \phi_{r}}= & \frac{1}{8}\left(\left(g_{Y X} g_{X}+g_{X}^{2}\right)\left(v_{d}^{2}-v_{u}^{2}\right)+2 g_{X}^{2}\left(v_{\eta}^{2}-v_{\bar{\eta}}^{2}\right)\right)+v_{\eta} v_{S} Y_{X} \lambda_{C} \\
& +m_{\tilde{\nu}}^{2}+\frac{1}{2} v_{u}^{2}\left|Y_{\nu}\right|^{2}+v_{\bar{\eta}}\left(2 v_{\bar{\eta}} Y_{X} Y_{X}+\sqrt{2} T_{X}\right) .
\end{aligned}
$$

To obtain the masses of sneutrinos, we use $Z^{R}$ to diagonalize $M_{\tilde{\nu}^{R}}^{2}$.

The mass matrix for CP-odd sneutrino $\left(\sigma_{l}, \sigma_{r}\right)$ is also deduced here

$$
\begin{aligned}
M_{\tilde{\nu}^{I}}^{2}= & \left(\begin{array}{cc}
m_{\sigma_{l} \sigma_{l}} & m_{\sigma_{r} \sigma_{l}}^{T} \\
m_{\sigma_{l} \sigma_{r}} & m_{\sigma_{r} \sigma_{r}}
\end{array}\right) \\
m_{\sigma_{l} \sigma_{l}}= & \frac{1}{8}\left(\left(g_{1}^{2}+g_{Y X}^{2}+g_{2}^{2}+g_{Y X} g_{X}\right)\left(v_{d}^{2}-v_{u}^{2}\right)+2 g_{Y X} g_{X}\left(v_{\eta}^{2}-v_{\bar{\eta}}^{2}\right)\right) \\
& \quad+\frac{1}{2} v_{u}^{2} Y_{\nu}^{T} Y_{\nu}+m_{\tilde{L}}^{2},
\end{aligned}
$$




$$
\begin{aligned}
m_{\sigma_{l} \sigma_{r}}= & \frac{1}{\sqrt{2}} v_{u} T_{\nu}-v_{u} v_{\bar{\eta}} Y_{X} Y_{\nu}-\frac{1}{2} v_{d}\left(\lambda_{H} v_{S}+\sqrt{2} \mu\right) Y_{\nu} \\
m_{\sigma_{r} \sigma_{r}}= & \frac{1}{8}\left(\left(g_{Y X} g_{X}+g_{X}^{2}\right)\left(v_{d}^{2}-v_{u}^{2}\right)+2 g_{X}^{2}\left(v_{\eta}^{2}-v_{\bar{\eta}}^{2}\right)\right)-v_{\eta} v_{S} Y_{X} \lambda_{C} \\
& +m_{\tilde{\nu}}^{2}+\frac{1}{2} v_{u}^{2}\left|Y_{\nu}\right|^{2}+v_{\bar{\eta}}\left(2 v_{\bar{\eta}} Y_{X} Y_{X}-\sqrt{2} T_{X}\right) .
\end{aligned}
$$

Using the matrix $Z^{I}$, we can diagonalize the mass squared matrix of the sneutrino $M_{\tilde{\nu}^{I}}^{2}$. In the same way, we deduce the mass matrixes for slepton and neutralino, and show them in the appendix.

Here, we show some needed couplings in this model. The CP-odd Higgs bosons interact with $\tilde{\nu}^{I}$ and $\tilde{\nu}^{R}$, whose concrete form is

$$
\begin{aligned}
\mathcal{L}_{A \tilde{\nu}^{I} \tilde{\nu}^{R}}= & A_{i} \tilde{\nu}_{j}^{I} \frac{i}{4} \sum_{a, b=1}^{3}\left\{\left[2 v_{S} \lambda_{C} Z_{k 3+b}^{R *} Z_{j 3+a}^{I *}\left(Y_{X}\right)_{a b} Z_{i 3}^{A}-2 \sqrt{2} Z_{k b}^{R *} Z_{j 3+a}^{I *}\left(T_{\nu}\right)_{a b} Z_{i 2}^{A}\right.\right. \\
& \left.\left.-2 \sqrt{2} Z_{k 3+b}^{R *} Z_{j 3+a}^{I *}\left(T_{X}\right)_{a b} Z_{i 4}^{A}+2 v_{\eta} \lambda_{C} Z_{k 3+b}^{R *} Z_{j 3+a}^{I *}\left(Y_{X}\right)_{a b} Z_{i 5}^{A}\right]+[R \leftrightarrow I, j \leftrightarrow k]\right\} \tilde{\nu}_{k}^{* R} .
\end{aligned}
$$

We also deduce the vertexes of $\tilde{\nu}_{k}^{R}-\bar{e}_{i}-\chi_{j}^{-}$and $\tilde{\nu}_{k}^{R}-\nu_{i}-\bar{\chi}_{i}^{0}$,

$$
\begin{aligned}
\mathcal{L}_{\tilde{\nu}^{R} \bar{e} \chi^{-}}= & \bar{e}_{i}\left\{\frac{i}{\sqrt{2}} U_{j 2}^{*} Z_{k i}^{R *} Y_{e}^{i} P_{L}-\frac{i}{\sqrt{2}} g_{2} V_{j 1} Z_{k i}^{R *} P_{R}\right\} \chi_{j}^{-} \tilde{\nu}_{k}^{R}, \\
\mathcal{L}_{\tilde{\nu}^{R} \nu \bar{\chi}^{0}}= & \bar{\chi}_{i}^{0}\left\{\frac{i}{2}\left(-g_{2} Z_{i 2}^{N *}+g_{Y X} Z_{i 5}^{N *}+g_{1} Z_{i 1}^{N *}\right) \sum_{a=1}^{3} Z_{k a}^{R *} U_{j a}^{V *} P_{L}\right. \\
& \left.+\frac{i}{2}\left(-g_{2} Z_{i 2}^{N}+g_{Y X} Z_{i 5}^{N}+g_{1} Z_{i 1}^{N}\right) \sum_{a=1}^{3} Z_{k a}^{R *} U_{j a}^{V} P_{R}\right\} \nu_{i} \tilde{\nu}_{k}^{R} .
\end{aligned}
$$

To save space in the text, the remaining vertexes are placed in appendix.

\section{Relic density}

In this section, we suppose the lightest mass eigenstate $\left(\tilde{\nu}_{1}^{R}\right)$ of CP-even sneutrino mass squared matrix in eq. (2.23) as a dark matter candidate and calculate the relic density. Any WIMP candidate has to satisfy the relic density constraints. The $\tilde{\nu}_{1}^{R}$ number density $n_{\tilde{\nu}_{1}^{R}}$ is governed by the Boltzmann equation [3, 51-53]

$$
\frac{d n_{\tilde{\nu}_{1}^{R}}}{d t}=-3 H n_{\tilde{\nu}_{1}^{R}}-\langle\sigma v\rangle_{S A}\left(n_{\tilde{\nu}_{1}^{R}}^{2}-n_{\tilde{\nu}_{1}^{R} e q}^{2}\right)-\langle\sigma v\rangle_{C A}\left(n_{\tilde{\nu}_{1}^{R}} n_{\phi}-n_{\tilde{\nu}_{1}^{R} e q} n_{\phi e q}\right) .
$$

$\tilde{\nu}_{1}^{R}$ can both self-annihilate and co-annihilate with another specy $\phi$. When the annihilation rate of $\tilde{\nu}_{1}^{R}$ becomes roughly equal to the Hubble expansion rate, the species freeze out at the temperature $T_{F}$,

$$
\langle\sigma v\rangle_{S A} n_{\tilde{\nu}_{1}^{R}}+\langle\sigma v\rangle_{C A} n_{\phi} \sim H\left(T_{F}\right)
$$


With the supposition $M_{\phi}>M_{\tilde{\nu}_{1}^{R}}[54]$

$$
n_{\phi}=\left(\frac{M_{\phi}}{M_{\tilde{\nu}_{1}^{R}}}\right)^{3 / 2} \operatorname{Exp}\left[\left(M_{\tilde{\nu}_{1}^{R}}-M_{\phi}\right) / T\right] n_{\tilde{\nu}_{1}^{R}}
$$

Then it becomes

$$
\left[\langle\sigma v\rangle_{S A}+\langle\sigma v\rangle_{C A}\left(\frac{M_{\phi}}{M_{\tilde{\nu}_{1}^{R}}}\right)^{3 / 2} \operatorname{Exp}\left[\left(M_{\tilde{\nu}_{1}^{R}}-M_{\phi}\right) / T\right]\right] n_{\tilde{\nu}_{1}^{R}} \sim H\left(T_{F}\right) .
$$

We study its annihilation rate $\langle\sigma v\rangle_{S A}\left(\langle\sigma v\rangle_{C A}\right)$ and its relic density $\Omega_{D}$ in the thermal history of the universe. To this end, the self-annihilation cross section $\sigma\left(\tilde{\nu}_{1}^{R} \tilde{\nu}_{1}^{R *} \rightarrow\right.$ anything) and co-annihilation cross section $\sigma\left(\tilde{\nu}_{1}^{R} \phi \rightarrow\right.$ anything) should be calculated. In the center of mass frame, their results can be written as $\sigma v_{\text {rel }}=a+b v_{\text {rel }}^{2}$, with $v_{\text {rel }}$ denoting the relative velocity of the two particles in the initial states. It is a good approximation to calculate the freeze-out temperature $\left(T_{F}\right)$ from the following formula $[3,53,55,56]$

$$
x_{F}=\frac{m_{D}}{T_{F}} \simeq \ln \left[\frac{0.038 M_{\mathrm{PL}} m_{D}\left(a+6 b / x_{F}\right)}{\sqrt{g_{*} x_{F}}}\right] .
$$

$M_{\mathrm{PL}}$ is the Planck mass $1.22 \times 10^{19} \mathrm{GeV} . m_{D}=m_{\tilde{\nu}_{1}^{R}}$ denoting the WIMP mass and $x_{F} \equiv m_{D} / T_{F} . g_{*}$ is the number of the relativistic degrees of freedom with mass less than $T_{F}$. The formula for the density of cold non-baryonic matter can be simplified in the following form $[3,9,53,57]$

$$
\Omega_{D} h^{2} \simeq \frac{1.07 \times 10^{9} x_{F}}{\sqrt{g_{*}} M_{\mathrm{PL}}\left(a+3 b / x_{F}\right) \mathrm{GeV}}
$$

and its value should be $\Omega_{D} h^{2}=0.1186 \pm 0.0020$ [13]

The dominant processes for the self-annihilation are: $\tilde{\nu}_{1}^{R}+\tilde{\nu}_{1}^{R} \rightarrow\{(W+W),(Z+$ $\left.Z),(h+h),\left(\bar{u}_{i}+u_{i}\right),\left(\bar{d}_{i}+d_{i}\right),\left(\bar{l}_{i}+l_{i}\right),\left(\bar{\nu}_{i}+\nu_{i}\right)\right\}$ with $i=1,2,3, h$ representing the lightest CP-even Higgs. $\nu_{i}$ denote three light neutrinos. The studied co-annihilation processes read as:

a. $\tilde{\nu}_{1}^{R}+\tilde{\nu}_{k}^{R} \rightarrow\left\{(W+W),(Z+Z),(h+h),\left(\bar{u}_{i}+u_{i}\right),\left(\bar{d}_{i}+d_{i}\right),\left(\bar{l}_{i}+l_{i}\right),\left(\bar{\nu}_{i}+\nu_{i}\right)\right\}$ with $k=2 \ldots 6, i=1,2,3$.

b. $\tilde{\nu}_{1}^{R}+\tilde{\nu}_{j}^{I} \rightarrow\left\{(W+W),(Z+h),\left(\bar{u}_{i}+u_{i}\right),\left(\bar{d}_{i}+d_{i}\right),\left(\bar{l}_{i}+l_{i}\right),\left(\bar{\nu}_{i}+\nu_{i}\right)\right\}$ and $j=$ $1 \ldots 6, i=1 \ldots 3$.

c. $\tilde{\nu}_{1}^{R}+\chi_{n}^{0} \rightarrow\left\{\left(Z+\nu_{i}\right),\left(W^{+}+l_{i}^{-}\right),\left(W^{-}+l_{i}^{+}\right)\right\}$and $n=1 \ldots 8, i=1 \ldots 3$.

\section{Direct detection}

The main scattering processes of CP-even sneutrinos off nucleons are $\tilde{\nu}^{R}+q \rightarrow \tilde{\nu}^{R}+q$ and $\tilde{\nu}^{R}+q \rightarrow \tilde{\nu}^{I}+q$. For the first type process $\tilde{\nu}^{R}+q \rightarrow \tilde{\nu}^{R}+q$, the exchanged particles 
are CP-even Higgs. While, for the second type process $\tilde{\nu}^{R}+q \rightarrow \tilde{\nu}^{I}+q$, the exchanged particles are vector bosons $Z$ and $Z^{\prime}$. The CP-odd Higgs boson contributions are much smaller than the contributions from CP-even Higgs boson and can be neglected safely [58]. After some calculation, we obtain the operators $\tilde{\nu}^{R *} \tilde{\nu}^{R} \bar{q} q$ and $\tilde{\nu}^{R *} \partial_{\mu} \tilde{\nu}^{R} \bar{q} \gamma^{\mu} q$ at the quark level.

To get the final results, we should convert the quark level coupling to the effective nucleon coupling. For the operator $\tilde{\nu}^{R *} \tilde{\nu}^{R} \bar{q} q$, the useful expressions are shown below [58]

$$
\begin{aligned}
a_{q} m_{q} \bar{q} q & \rightarrow f_{N} m_{N} \bar{N} N, & f_{N} & =\sum_{q=u, d, s} f_{T q}^{(N)} a_{q}+\frac{2}{27} f_{T G}^{(N)} \sum_{q=c, b, t} a_{q}, \\
\left\langle N\left|m_{q} \bar{q} q\right| N\right\rangle & =m_{N} f_{T q}^{(N)}, & f_{T G}^{(N)} & =1-\sum_{q=u, d, s} f_{T q}^{(N)} .
\end{aligned}
$$

$f_{N}$ includes the coupling to gluons induced by integrating out heavy quark loops. The numbers of $f_{T q}^{(N)}$ are collected here [59-61],

$$
\begin{aligned}
& f_{T u}^{(p)}=0.0153, \quad f_{T d}^{(p)}=0.0191, \quad f_{T s}^{(p)}=0.0447, \\
& f_{T u}^{(n)}=0.0110, \quad f_{T d}^{(n)}=0.0273, \quad f_{T s}^{(n)}=0.0447 .
\end{aligned}
$$

It is easy to convert the operator $b_{q} \tilde{\nu}^{R *} \partial_{\mu} \tilde{\nu}^{R} \bar{q} \gamma^{\mu} q$ to $b_{N} \tilde{\nu}^{R *} \partial_{\mu} \tilde{\nu}^{R} \bar{N} \gamma^{\mu} N$ through the following formulas

$$
b_{p}=2 b_{u}+b_{d}, \quad b_{n}=2 b_{d}+b_{u}
$$

With the obtained $f_{N}$, one gets the scattering cross section

$$
\sigma=\frac{1}{\pi} \mu^{2}\left[Z_{p} f_{p}+\left(A-Z_{p}\right) f_{n}\right]^{2}
$$

Here $Z_{p}$ is the number of proton, and $A$ represents the number of atom.

\section{Numerical results}

In this section, we study the numerical results. $Z^{\prime}$ boson properties are constrained by manifold low energy experiments [62,63]. The lower limits on the mass of $Z^{\prime}$ set by low energy data are about $1 \mathrm{TeV}$ in some models. The mass bounds for $M_{Z^{\prime}}$ from LHC are about several $\mathrm{TeV}$, which are more severe than those from low energy constraints. In the case of final states with taus, the lower mass limits for $Z^{\prime}$ obtained at $13 \mathrm{TeV}$ are as high as $\sim 2.4 \mathrm{TeV}$ [64]. Another stringent for the mass of $Z^{\prime}$ is set in the fully hadronic channel, with a lower mass limit of $2.35 \mathrm{TeV}$ in the context of the Heavy Vector Triplet model weakly -coupled scenario A [65]. The result from ATLAS collaboration at $\sqrt{s}=13 \mathrm{TeV}$ obtained with 2016 data is more stringent [66]. The resulting 95\% CL lower mass limits are $4.5 \mathrm{TeV}$ for the $Z_{\mathrm{SSM}}^{\prime}$ in the Sequential Standard Model, $4.1 \mathrm{TeV}$ for the $Z_{\chi}^{\prime}$, and $3.8 \mathrm{TeV}$ for the $Z_{\psi}^{\prime}$. Here, $Z_{\chi}^{\prime}$ and $Z_{\psi}^{\prime}$ belong to the $E_{6}$-motivated model. Other $E_{6} Z^{\prime}$ models are also constrained in the range between those quoted for the $Z_{\chi}^{\prime}$ and $Z_{\psi}^{\prime}$. The lower mass limits 
are $4.1 \mathrm{TeV}$ for the $Z_{3 R}^{\prime}$ in the left-right symmetric model, and $4.2 \mathrm{TeV}$ for the $Z_{B-L}^{\prime}$ of the $(B-L)$ model [66]. The authors $[67,68]$ give the upper bound $\left(M_{Z^{\prime}} / g_{X} \geq 6 \mathrm{TeV}\right)$ on the ratio between $M_{Z^{\prime}}$ and its gauge coupling at $99 \% \mathrm{CL} \cdot \tan \beta_{\eta}$ is also constrained by the LHC experimental data and should be smaller than 1.5 [69]. In order to satisfy the constraints from LHC, we choose the parameters to make $M_{Z^{\prime}}>4.5 \mathrm{TeV}$, because the quoted number are valid in other models and do not apply directly. The constraints for supersymmetric particles, shown in ref. [13], are also taken into account.

Considering the above constraints, we use the following parameters

$$
\begin{aligned}
M_{S} & =0.8 \mathrm{TeV}, \quad T_{\kappa}=1.6 \mathrm{TeV}, \quad M_{1}=M_{2}=M_{B L}=1 \mathrm{TeV}, \quad \tan \beta=11, \quad g_{Y X}=0.2, \\
v_{\eta} & =15.5 \times \cos \beta_{\eta} \mathrm{TeV}, \quad v_{\bar{\eta}}=15.5 \times \sin \beta_{\eta} \mathrm{TeV}, \quad Y_{X 11}=Y_{X 22}=0.5, \quad Y_{X 33}=0.4, \\
g_{X} & =\kappa=\lambda_{H}=0.3, \quad \lambda_{C}=-0.3, \quad M_{Q}^{2}=2.5 \mathrm{TeV}^{2}, \quad M_{B B^{\prime}}=0.4 \mathrm{TeV}, \quad T_{\lambda_{H}}=1.8 \mathrm{TeV}, \\
T_{X 11} & =T_{X 22}=-1 \mathrm{TeV}, \quad T_{X 33}=-2 \mathrm{TeV}, \quad T_{e 11}=T_{e 22}=-3 \mathrm{TeV}, \quad T_{e 33}=-4 \mathrm{TeV}, \\
l_{W} & =4 \mathrm{TeV}^{2}, \quad B_{\mu}=B_{S}=M_{T}^{2}=1 \mathrm{TeV}^{2}, \quad \tan \beta_{\eta}=0.83, \quad T_{\nu 11}=T_{\nu 22}=0, \quad A_{t}=2.6 \mathrm{TeV}, \\
T_{\lambda_{C}} & =0.25 \mathrm{TeV}, \quad M_{\nu 11}^{2}=M_{\nu 22}^{2}=0.5 \mathrm{TeV}^{2}, \quad M_{L 11}^{2}=M_{L 22}^{2}=M_{E 11}^{2}=M_{E 22}^{2}=3 \mathrm{TeV}^{2} .
\end{aligned}
$$

Here, we take $T_{\nu}, T_{X}$ and $M_{\nu}$ as diagonal matrices, for example

$$
T_{X}=\left(\begin{array}{ccc}
T_{X 11} & 0 & 0 \\
0 & T_{X 22} & 0 \\
0 & 0 & T_{X 33}
\end{array}\right) .
$$

We list the remaining parameters which will vary in the following numerical analysis:

$$
v_{S}, \quad \mu, T_{\nu 33}, \quad M_{L 33}^{2}, M_{\nu 33}^{2}, \quad M_{E 33}^{2}, m_{S}^{2} .
$$

Firstly, we research the lightest CP-even Higgs mass including the loop corrections and discuss the other CP-even Higgs masses. Secondly, the relic density of the lightest $\mathrm{CP}$-even sneutrino is calculated numerically. At last, we study the cross section for the lightest sneutrino scattering off nucleon.

\subsection{Higgs mass}

Considering the loop corrections from top and stop contributions, we study the SM-like Higgs boson mass in this subsection. For simplicity, we suppose that $\mu=0.5 \mathrm{TeV}$ and $m_{S}^{2}=1 \mathrm{TeV}^{2}$ in the following analysis. $v_{S}$ is the VEV of $S$ and emerges in the diagonal elements of CP-even Higgs mass squared matrix (eqs. (A.1) and (A.2)). So, the lightest tree-level Higgs mass $m_{h_{1}}^{0}$ is the increasing function of $v_{S}$. More important, $v_{S}$ affects the lightest neutralino mass through the element $m_{\tilde{H}_{d}^{0} \tilde{H}_{u}^{0}}=-\frac{1}{\sqrt{2}} \lambda_{H} v_{S}-\mu$ in eq. (A.7). In our used parameter space, to keep $\tilde{\nu}_{1}^{R}$ as LSP we run $v_{S}$ from 2500 to $3500 \mathrm{GeV}$ and show $m_{h}$ varying with $v_{S}$ in figure 1 . The gray area is the lightest CP-even Higgs mass in $\pm 3 \sigma$ sensitivity band. Obviously, $m_{h}$ is the increasing function of $v_{S}$. In the $v_{S}$ region (2700-3300) GeV, $m_{h}$ can satisfy the experimental bound on the SM-like Higgs boson mass in $\pm 3 \sigma$ sensitivity. And the other CP-even Higgs boson masses are all heavier than $2.5 \mathrm{TeV}$ in this case. 


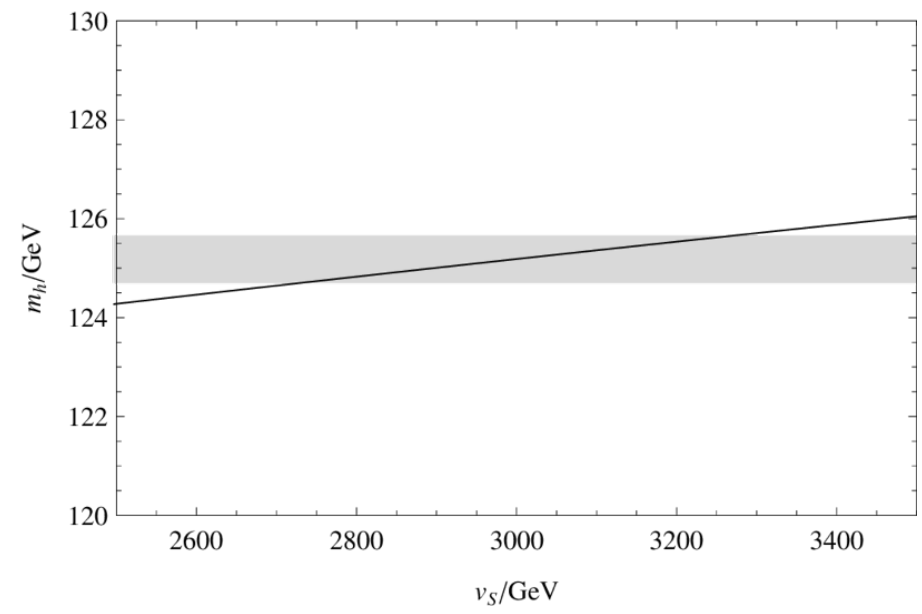

Figure 1. Considering the loop corrections, the lightest CP-even Higgs mass $\left(m_{h}\right)$ versus $v_{S}$ is plotted by the solid line with $A_{t}=2.6 \mathrm{TeV}$.

\subsection{Relic density of sneutrino dark matter}

Here, the parameters $v_{S}=3 \mathrm{TeV}$ and $m_{S}^{2}=1 \mathrm{TeV}^{2}$ are used to study the relic density of dark matter. With the same parameters, the lightest neutralino in the MSSM is around $500 \mathrm{GeV}$ as $\mu=500 \mathrm{GeV}$. When $|\mu|$ is near zero, the mass of the lightest neutralino in the MSSM is very tiny. However, the case in the $\mathrm{U}(1)_{X} \mathrm{SSM}$ is different from that in the MSSM. The neutralino mass matrix (eqs. (A.7)) in the U(1) ${ }_{X} \mathrm{SSM}$ is $8 \times 8$, where $m_{\tilde{H}_{d}^{0} \tilde{H}_{u}^{0}}=-\frac{1}{\sqrt{2}} \lambda_{H} v_{S}-\mu$ (eqs. (A.8)) corresponds to $-\mu$ in the neutralino mass matrix of MSSM. According to our parameters $v_{S}=3000 \mathrm{GeV}$ and $\lambda_{H}=0.3, m_{\tilde{H}_{d}^{0} \tilde{H}_{u}^{0}}=-\frac{1000}{\sqrt{2}} \mathrm{GeV}-$ $\mu \sim-707 \mathrm{GeV}-\mu$. That is to say, $m_{\tilde{H}_{d}^{0} \tilde{H}_{u}^{0}}$ is equal to the shift of $-\mu$. Therefore, as $\mu=0, m_{\tilde{H}_{d}^{0} \tilde{H}_{u}^{0}}$ is around $-707 \mathrm{GeV}$, and the lightest neutralino is around $650 \mathrm{GeV}$. The other terms in eqs. (A.7) slightly influence the lightest neutralino. When $\mu$ is during the region $(-400,-1100) \mathrm{GeV}$, the lightest neutralino will be small, but in this parameter space the corresponding relic density $\Omega_{D} h^{2}$ can not satisfy the non-baryonic density value. Considering these constraints, we plot $\Omega_{D} h^{2}$ versus $\mu$ in figure 2 with $\mu$ varying from 0 to $2000 \mathrm{GeV}$. The remaining parameters are $m_{\nu 33}^{2}=250^{2} \mathrm{GeV}^{2}, T_{\nu 33}=1.6 \mathrm{TeV}, M_{L 33}^{2}=$ $M_{E 33}^{2}=3 \mathrm{TeV}^{2}$. The gray area represents the relic density in $\pm 3 \sigma$ sensitivity band. In the $\mu$ region $(0,2000) \mathrm{GeV}$, the relic density is the decreasing function. From this diagram, one can find that as $\mu$ near $500 \mathrm{GeV}$ the result is close to the center value of the relic density. In $\pm 1 \sigma$ sensitivity of $\Omega_{D} h^{2}$, the lightest neutralino is around $850 \mathrm{GeV}$. The choice of parameters are chosen for illustration.

To more accurately scan the parameter space, the numerical results of the relic density in $\pm 3 \sigma$ sensitivity are plotted in the plane of $M_{L 33}^{2}$ and $T_{\nu 33}$ as $\mu=500 \mathrm{GeV}, m_{\nu 33}^{2}=$ $250^{2} \mathrm{GeV}^{2}, M_{E 33}^{2}=3 \mathrm{TeV}^{2} . \quad M_{L 33}^{2}$ and $T_{\nu 33}$ come from the soft breaking terms. As the non-diagonal element of sneutrino mass matrix, $T_{\nu 33}$ affects the sneutrino masses and mixing. On the other hand, $M_{L 33}^{2}$ appears in the diagonal elements of the mass matrixes for sneutrino and slepton. So, $M_{L 33}^{2}$ influences the both type scalars. The allowed results are plotted by the dots in figure 3 , where they are almost symmetric with respect to $T_{\nu 33}=0$. 


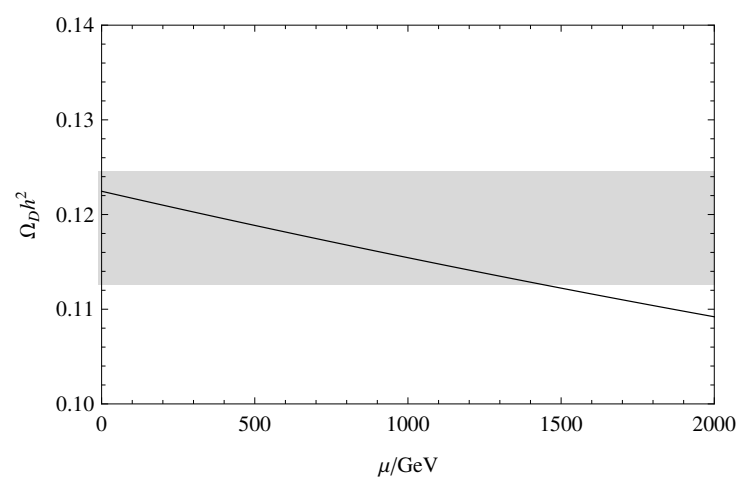

Figure 2. The relic density versus $\mu$.

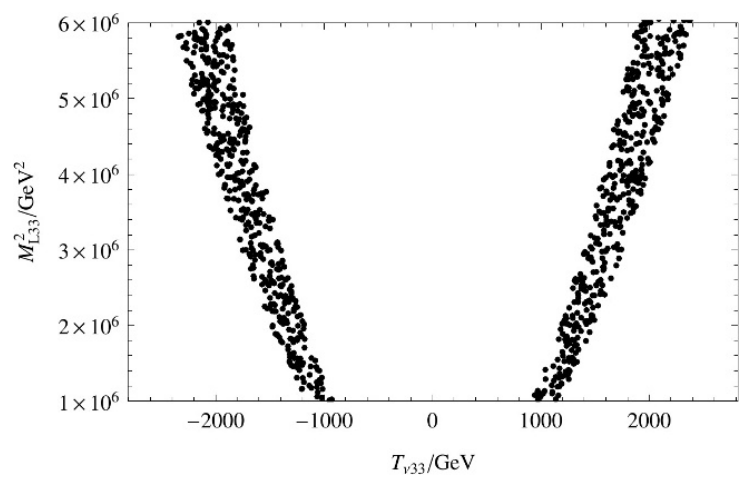

Figure 3. The allowed results of the relic density in the plane of $M_{L 33}^{2}$ and $T_{\nu 33}$.

In the plan of $M_{E 33}^{2}$ and $M_{\nu 33}^{2}$, the allowed results in $\pm 3 \sigma$ sensitivity of $\Omega_{D} h^{2}$ are also researched by taking $\mu=500 \mathrm{GeV}, T_{\nu 33}=1.6 \mathrm{TeV}$ and $M_{L 33}^{2}=3 \mathrm{TeV}^{2}$. We show these results by the dots in figure 4 . The effect of $M_{E 33}^{2}$ is small, because it influences the numerical results only by affecting the slepton mixing and masses. $M_{\nu 33}^{2}$ appears in the mass matrix of sneutrino, which can affect the lightest sneutrino mass and the mixing of sneutrino. Therefore, $M_{\nu 33}^{2}$ is a sensitive parameter, and has obvious influence on $m_{\tilde{\nu}_{1}^{R}}$ and $\Omega_{D} h^{2}$. The favorite region of $M_{\nu 33}^{2}$ is from 60000 to $68000 \mathrm{GeV}^{2}$. This region of $M_{\nu 33}^{2}$ can also keep the lightest CP-even sneutrino $\tilde{\nu}_{1}^{R}$ as LSP.

According to the parameter space under consideration, the lightest CP-even sneutrino mass is about $320 \mathrm{GeV}$. The other CP-even sneutrinos $\left(\tilde{\nu}_{2}^{R} \ldots \tilde{\nu}_{6}^{R}\right)$ are all heavier than $1900 \mathrm{GeV}$. The masses of all CP-odd sneutrinos $\left(\tilde{\nu}_{1}^{I} \ldots \tilde{\nu}_{6}^{I}\right)$ are larger than $1900 \mathrm{GeV}$. For the relic density in $\pm 1 \sigma$ sensitivity, the lightest neutralino is around $850 \mathrm{GeV}$. That is to say $\tilde{\nu}_{1}^{R}$ is the LSP, and can be the dark matter candidate.

If the mass of the virtual particle in s-channel is around $2 M_{D}$, the resonance annihilation will occur. The resonance annihilation strongly affects the annihilation crosssection hence the relic density. In these numerical results, the mass of dark matter is $M_{D} \sim 320 \mathrm{GeV}$. The four virtual CP-even Higgs bosons in s-channel are all heavier than $2.5 \mathrm{TeV}$, and the lightest CP-even Higgs boson is about $125 \mathrm{GeV}$. It is obvious that $2 M_{D}$ is far from all the CP-even Higgs masses. So the resonance annihilation can not take place. 


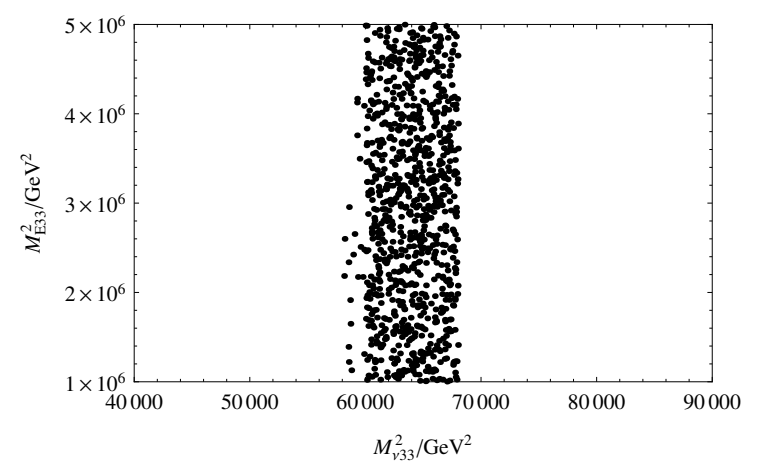

Figure 4. The allowed results of the relic density in the plane of $M_{E 33}^{2}$ and $M_{\nu 33}^{2}$.

\subsection{The cross section of the sneutrino scattering off nucleon}

Taking into account the constraint from the relic density, we calculate numerically the cross section of the sneutrino scattering off nucleon in this subsection. Within the considered parameter space, the lightest CP-even sneutrino is around $320 \mathrm{GeV}$. The experimental limit on direct detection for a dark matter of $320 \mathrm{GeV}$ is about $2.5 \times 10^{-46} \mathrm{~cm}^{2}$ for Xenon and about twice as large for PandaX [70,71]. Using the parameters $v_{S}=3 \mathrm{TeV}, m_{\nu 33}^{2}=$ $250^{2} \mathrm{GeV}^{2}, M_{L 33}^{2}=M_{E 33}^{2}=3 \mathrm{TeV}^{2}$ that can satisfy the relic density constraint, we research the cross section of the sneutrino scattering off nucleon.

$m_{S}^{2}$ is the mass square term of $S^{2}$ in the soft breaking terms. It does not have relation with the masses of sneutrinos and neutralinos. Because of the mixing of $\mathrm{S}$ and neutral CP-even Higgs (eqs. (A.1) and (A.2)), $m_{S}^{2}$ impacts the CP-even Higgs masses and Higgs mixing to some extent. $m_{S}^{2}$ can directly improve heavy Higgs mass, but its effect to the lightest CP-even Higgs mass $m_{h_{1}}^{0}$ at the tree-level is very small. CP-even Higgs bosons give dominant contribution to the relic density, so $m_{S}^{2}$ is constrained by $\Omega_{D} h^{2}$. Considering this constraint, we adopt $m_{S}^{2}$ region as $[0.6,2.0] \mathrm{TeV}^{2}$. In figure 5 , the cross section versus $m_{S}^{2}$ is plotted by the solid line with $\mu=500 \mathrm{GeV}$ and $T_{\nu 33}=1.6 \mathrm{TeV}$. The solid line is in the region $\left(6.5 \times 10^{-48}, 8.0 \times 10^{-48}\right) \mathrm{cm}^{2}$, when $m_{S}^{2}$ varies from 0.6 to $2 \mathrm{TeV}^{2}$. These results for $m_{D} \sim 320 \mathrm{GeV}$ are more than one order of magnitude below current limits.

To further discuss the sneutrino scattering off nucleon, in figure 6 we plot the cross section versus $\mu$ by the solid line (dotted line) with $T_{\nu 33}=1.6(1.4) \mathrm{TeV}$ and $m_{S}^{2}=3 \mathrm{TeV}^{2}$. As discussed for the figure 2 , to satisfy the constraints from $\Omega_{D} h^{2}$ and $\tilde{\nu}_{1}^{R}$ as the LSP, we take $\mu$ in the region $[0,2000] \mathrm{GeV}$. For the same $\mu$, the value of the solid line is a little bigger than the value of the dotted line. The solid line and dotted line possess similar behaviors and they are increasing functions of $\mu$. As $\mu=500 \mathrm{GeV}$, the solid line and dotted line are around $7 \times 10^{-48} \mathrm{~cm}^{2}$. While, the cross section can reach $10^{-47} \mathrm{~cm}^{2}$ with $\mu$ near $2000 \mathrm{GeV}$. In our parameter space, the theoretical predictions for this model for the benchmark chosen are smaller than the current limits by one order of magnitude.

\section{Discussion and conclusion}

The $\mathrm{U}(1)_{X} \mathrm{SSM}$ is the extension of MSSM, whose local gauge group is $\mathrm{SU}(3)_{C} \times \mathrm{SU}(2)_{L} \times$ $\mathrm{U}(1)_{Y} \times \mathrm{U}(1)_{X}$. To obtain this model, righ-handed neutrinos and three Higgs superfields 


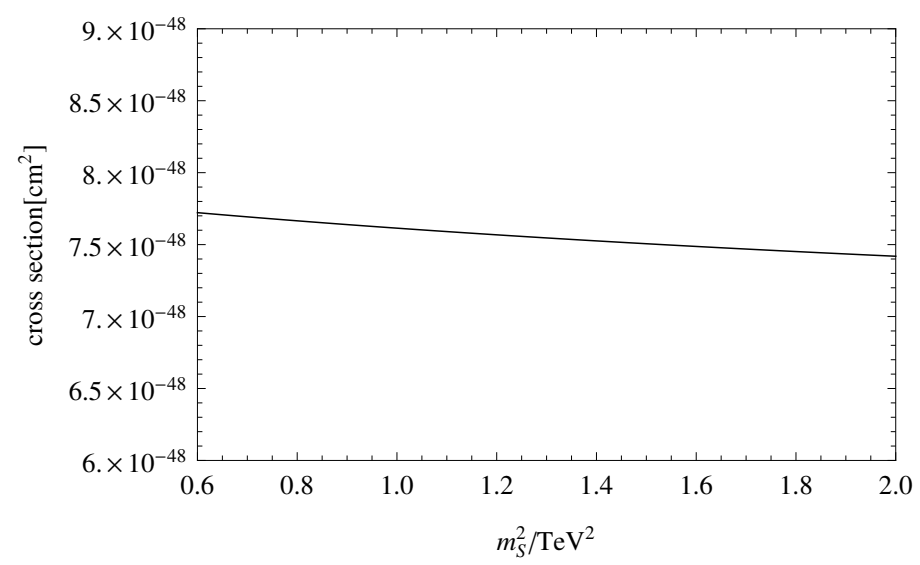

Figure 5. The cross section versus $m_{S}^{2}$.

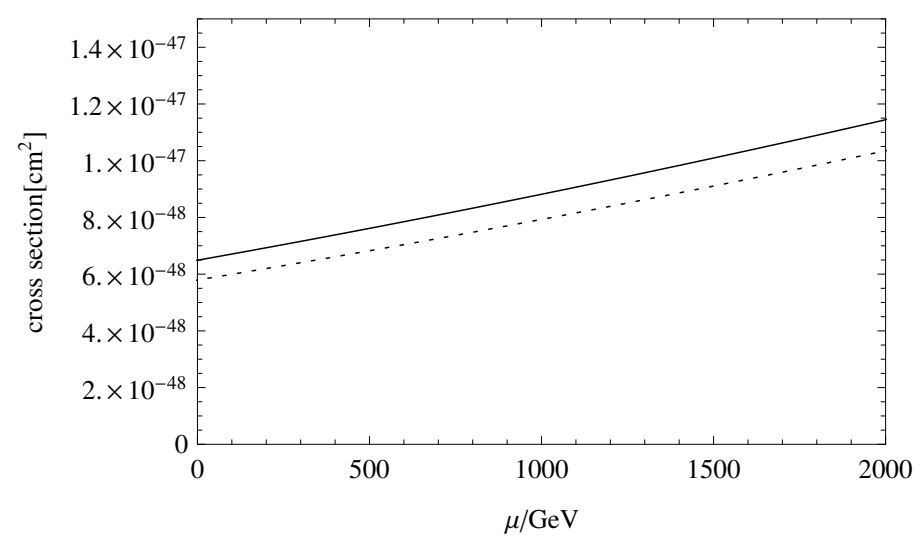

Figure 6. The cross section versus $\mu$ is plotted by solid line (dotted line) with $T_{\nu 33}=1.6$ (1.4) TeV.

$\hat{\eta}, \hat{\eta}, \hat{S}$ are added to the MSSM. Through the seesaw mechanism, three tiny neutrino masses can be produced. The right-handed sneutrinos are sterile, and if they are main parts of the lightest sneutrino, it possesses the characters of cold dark matter.

Taking into account the loop corrections, we study the lightest CP-even Higgs mass (SM-like) in the U(1) $)_{X}$ SSM. Comparing with the MSSM, there are three additional Higgs superfields $(\hat{\eta}, \hat{\eta}, \hat{S})$ in the $\mathrm{U}(1)_{X} \mathrm{SSM}$, which is also discussed. With the assumption that the lightest $\mathrm{CP}$-even sneutrino can be a cold dark matter candidate, the relic density of dark matter and the cross section of dark matter scattering off nucleon are both studied. The virtual Higgs contributions to both the relic density and the scattering cross section are dominant. The numerical results imply that the parameters $M_{\nu 33}^{2}, M_{L 33}^{2}, T_{\nu 33}$ and $\mu$ are all important. The used parameter space is reasonable and satisfy the dark matter constraints from both the relic density and the scattering off nucleon. This work gives constraints to the parameter space of the $\mathrm{U}(1)_{X} \mathrm{SSM}$ and may be benefit for the future direct detection. 


\section{Acknowledgments}

We are very grateful to Wei Chao the professor of Beijing Normal University for giving us some useful discussions and Tiago Adorno the professor of Hebei University for English rewriting. This work is supported by National Natural Science Foundation of China (NNSFC) (No. 11535002, No. 11605037, No. 11705045), Post-graduate's Innovation Fund Project of Hebei Province (No. CXZZBS2019027), Hebei Key Lab of Optic-Electronic Information and Materials, and the youth top-notch talent support program of the Hebei Province.

\section{A Mass matrix}

In the basis $\left(\phi_{d}, \phi_{u}, \phi_{\eta}, \phi_{\bar{\eta}}, \phi_{s}\right)$, the mass squared matrix of CP-even Higgs reads

$$
m_{h}^{2}=\left(\begin{array}{ccccc}
m_{\phi_{d} \phi_{d}} & m_{\phi_{u} \phi_{d}} & m_{\phi_{\eta} \phi_{d}} & m_{\phi_{\bar{\eta}} \phi_{d}} & m_{\phi_{s} \phi_{d}} \\
m_{\phi_{d} \phi_{u}} & m_{\phi_{u} \phi_{u}} & m_{\phi_{\eta} \phi_{u}} & m_{\phi_{\bar{\eta}} \phi_{u}} & m_{\phi_{s} \phi_{u}} \\
m_{\phi_{d} \phi_{\eta}} & m_{\phi_{u} \phi_{\eta}} & m_{\phi_{\eta} \phi_{\eta}} & m_{\phi_{\bar{\eta}} \phi_{\eta}} & m_{\phi_{s} \phi_{\eta}} \\
m_{\phi_{d} \phi_{\bar{\eta}}} & m_{\phi_{u} \phi_{\bar{\eta}}} & m_{\phi_{\eta} \phi_{\bar{\eta}}} & m_{\phi_{\bar{\eta}} \phi_{\bar{\eta}}} & m_{\phi_{s} \phi_{\bar{\eta}}} \\
m_{\phi_{d} \phi_{s}} & m_{\phi_{u} \phi_{s}} & m_{\phi_{\eta} \phi_{s}} & m_{\phi_{\bar{\eta}} \phi_{s}} & m_{\phi_{s} \phi_{s}}
\end{array}\right) .
$$

The explicit forms of the elements $m_{\phi_{d} \phi_{d}}$ etc. in this mass matrix are shown

$$
\begin{aligned}
m_{\phi_{d} \phi_{d}}= & m_{H_{d}}^{2}+|\mu|^{2}+\frac{1}{8}\left(\left[g_{1}^{2}+\left(g_{X}+g_{Y X}\right)^{2}+g_{2}^{2}\right]\left(3 v_{d}^{2}-v_{u}^{2}\right)\right. \\
& \left.+2\left(g_{Y X} g_{X}+g_{X}^{2}\right)\left(v_{\eta}^{2}-v_{\bar{\eta}}^{2}\right)\right)+\sqrt{2} v_{S} \mu \lambda_{H}+\frac{1}{2}\left(v_{u}^{2}+v_{S}^{2}\right)\left|\lambda_{H}\right|^{2}, \\
m_{\phi_{d} \phi_{u}}= & -\frac{1}{4}\left(g_{2}^{2}+\left(g_{Y X}+g_{X}\right)^{2}+g_{1}^{2}\right) v_{d} v_{u}+\left|\lambda_{H}\right|^{2} v_{d} v_{u}-\lambda_{H} l_{W} \\
& -\frac{1}{2} \lambda_{H}\left(v_{\eta} v_{\bar{\eta}} \lambda_{C}+v_{S}^{2} \kappa\right)-B_{\mu}-\sqrt{2} v_{S}\left(\frac{1}{2} T_{\lambda_{H}}+M_{S} \lambda_{H}\right), \\
m_{\phi_{u} \phi_{u}}= & m_{H_{u}}^{2}+|\mu|^{2}+\frac{1}{8}\left(\left[g_{1}^{2}+\left(g_{X}+g_{Y X}\right)^{2}+g_{2}^{2}\right]\left(3 v_{u}^{2}-v_{d}^{2}\right)\right. \\
& \left.+2\left(g_{Y X} g_{X}+g_{X}^{2}\right)\left(v_{\bar{\eta}}^{2}-v_{\eta}^{2}\right)\right)+\sqrt{2} v_{S} \mu \lambda_{H}+\frac{1}{2}\left(v_{d}^{2}+v_{S}^{2}\right)\left|\lambda_{H}\right|^{2}, \\
m_{\phi_{d} \phi_{\eta}}= & \frac{1}{2} g_{X}\left(g_{Y X}+g_{X}\right) v_{d} v_{\eta}-\frac{1}{2} v_{u} v_{\bar{\eta}} \lambda_{H} \lambda_{C}, \\
m_{\phi_{u} \phi_{\eta}=}= & -\frac{1}{2} g_{X}\left(g_{Y X}+g_{X}\right) v_{u} v_{\eta}-\frac{1}{2} v_{d} v_{\bar{\eta}} \lambda_{H} \lambda_{C}, \\
m_{\phi_{\eta} \phi_{\eta}}= & m_{\eta}^{2}+\frac{1}{4}\left(\left(g_{Y X} g_{X}+g_{X}^{2}\right)\left(v_{d}^{2}-v_{u}^{2}\right)+2 g_{X}^{2}\left(3 v_{\eta}^{2}-v_{\bar{\eta}}^{2}\right)\right)+\frac{\left|\lambda_{C}\right|^{2}}{2}\left(v_{\bar{\eta}}^{2}+v_{S}^{2}\right), \\
m_{\phi_{d} \phi_{\bar{\eta}}}= & -\frac{1}{2} g_{X}\left(g_{Y X}+g_{X}\right) v_{d} v_{\bar{\eta}}-\frac{1}{2} v_{u} v_{\eta} \lambda_{H} \lambda_{C}, \\
m_{\phi_{u} \phi_{\bar{\eta}}}= & \frac{1}{2} g_{X}\left(g_{Y X}+g_{X}\right) v_{u} v_{\bar{\eta}}-\frac{1}{2} v_{d} v_{\eta} \lambda_{H} \lambda_{C},
\end{aligned}
$$




$$
\begin{aligned}
& m_{\phi_{\eta} \phi_{\bar{\eta}}}=-g_{X}^{2} v_{\eta} v_{\bar{\eta}}+\frac{1}{2}\left(2 l_{W}-\lambda_{H} v_{d} v_{u}\right) \lambda_{C}+\left|\lambda_{C}\right|^{2} v_{\eta} v_{\bar{\eta}} \\
&+\frac{1}{\sqrt{2}} v_{S}\left(2 M_{S} \lambda_{C}+T_{\lambda_{C}}\right)+\frac{1}{2} v_{S}^{2} \lambda_{C} \kappa \\
& m_{\phi_{\bar{\eta}} \phi_{\bar{\eta}}}= m_{\bar{\eta}}^{2}+\frac{1}{4}\left(\left(g_{Y X} g_{X}+g_{X}^{2}\right)\left(v_{u}^{2}-v_{d}^{2}\right)+2 g_{X}^{2}\left(3 v_{\bar{\eta}}^{2}-v_{\eta}^{2}\right)\right)+\frac{\left|\lambda_{C}\right|^{2}}{2}\left(v_{\eta}^{2}+v_{S}^{2}\right), \\
& m_{\phi_{d} \phi_{s}}=\left(\lambda_{H} v_{d} v_{S}+\sqrt{2} v_{d} \mu-v_{u}\left(\kappa v_{S}+\sqrt{2} M_{S}\right)\right) \lambda_{H}-\frac{1}{\sqrt{2}} v_{u} T_{\lambda_{H}}, \\
& m_{\phi_{u} \phi_{s}}=\left(\lambda_{H} v_{u} v_{S}+\sqrt{2} v_{u} \mu-v_{d}\left(\kappa v_{S}+\sqrt{2} M_{S}\right)\right) \lambda_{H}-\frac{1}{\sqrt{2}} v_{d} T_{\lambda_{H}}, \\
& m_{\phi_{\eta} \phi_{s}}=\left(\lambda_{C} v_{\eta} v_{S}+v_{\bar{\eta}}\left(\kappa v_{S}+\sqrt{2} M_{S}\right)\right) \lambda_{C}+\frac{1}{\sqrt{2}} v_{\bar{\eta}} T_{\lambda_{C}}, \\
& m_{\phi_{\bar{\eta}} \phi_{s}}=\left(\lambda_{C} v_{\bar{\eta}} v_{S}+v_{\eta}\left(\kappa v_{S}+\sqrt{2} M_{S}\right)\right) \lambda_{C}+\frac{1}{\sqrt{2}} v_{\eta} T_{\lambda_{C}}, \\
& m_{\phi_{s} \phi_{s}}= m_{S}^{2}+\left(2 l_{W}+3 v_{S}\left(\kappa v_{S}+2 \sqrt{2} M_{S}\right)+\lambda_{C} v_{\eta} v_{\bar{\eta}}-\lambda_{H} v_{d} v_{u}\right) \kappa \\
&+\frac{1}{2}\left|\lambda_{C}\right|^{2} \xi^{2}+\frac{1}{2}\left|\lambda_{H}\right|^{2} v^{2}+2 B_{S}+4\left|M_{S}\right|^{2}+\sqrt{2} v_{S} T_{\kappa} . \\
&\left(\begin{array}{l}
m_{\sigma_{d} \sigma_{d}} m_{\sigma_{u} \sigma_{d}} m_{\sigma_{\eta} \sigma_{d}} m_{\sigma_{\bar{\eta}} \sigma_{d}} m_{\sigma_{s} \sigma_{d}} \\
m_{\sigma_{d} \sigma_{u}} m_{\sigma_{u} \sigma_{u}} m_{\sigma_{\eta} \sigma_{u}} m_{\sigma_{\bar{\eta}} \sigma_{u}} m_{\sigma_{s} \sigma_{u}} \\
m_{\sigma_{d} \sigma_{\eta}} m_{\sigma_{u} \sigma_{\eta}} m_{\sigma_{\eta} \sigma_{\eta}} m_{\sigma_{\bar{\eta}} \sigma_{\eta}} m_{\sigma_{s} \sigma_{\eta}} \\
m_{\sigma_{d} \sigma_{\bar{\eta}}} m_{\sigma_{u} \sigma_{\bar{\eta}}} m_{\sigma_{\eta} \sigma_{\bar{\eta}}} m_{\sigma_{\bar{\eta}} \sigma_{\bar{\eta}}} m_{\sigma_{s} \sigma_{\bar{\eta}}} \\
m_{\sigma_{d} \sigma_{s}} m_{\sigma_{u} \sigma_{s}} m_{\sigma_{\eta} \sigma_{s}} m_{\sigma_{\bar{\eta}} \sigma_{s}} m_{\sigma_{s} \sigma_{s}}
\end{array}\right) . \\
& m_{A^{0}}^{2}=
\end{aligned}
$$

Eq. (A.3) is the CP-odd Higgs mass squared matrix, whose elements are

$$
\begin{aligned}
m_{\sigma_{d} \sigma_{d}}= & m_{H_{d}}^{2}+|\mu|^{2}+\frac{1}{8}\left(\left[g_{1}^{2}+\left(g_{X}+g_{Y X}\right)^{2}+g_{2}^{2}\right]\left(v_{d}^{2}-v_{u}^{2}\right)\right. \\
& \left.+2\left(g_{Y X} g_{X}+g_{X}^{2}\right)\left(v_{\eta}^{2}-v_{\bar{\eta}}^{2}\right)\right)+\sqrt{2} v_{S} \mu \lambda_{H}+\frac{1}{2}\left(v_{u}^{2}+v_{S}^{2}\right)\left|\lambda_{H}\right|^{2}, \\
m_{\sigma_{d} \sigma_{u}}= & \left(\left(\sqrt{2} M_{S} v_{S}+l_{W}\right)+\frac{1}{2} \kappa v_{S}^{2}+\frac{1}{2} \lambda_{C} v_{\eta} v_{\bar{\eta}}\right) \lambda_{H}+B_{\mu}+\frac{1}{\sqrt{2}} v_{S} T_{\lambda_{H}}, \\
m_{\sigma_{u} \sigma_{u}}= & m_{H_{u}}^{2}+|\mu|^{2}+\frac{1}{8}\left(\left[g_{1}^{2}+\left(g_{X}+g_{Y X}\right)^{2}+g_{2}^{2}\right]\left(v_{u}^{2}-v_{d}^{2}\right)\right. \\
& \left.+2\left(g_{Y X} g_{X}+g_{X}^{2}\right)\left(v_{\bar{\eta}}^{2}-v_{\eta}^{2}\right)\right)+\sqrt{2} v_{S} \mu \lambda_{H}+\frac{1}{2}\left(v_{d}^{2}+v_{S}^{2}\right)\left|\lambda_{H}\right|^{2}, \\
m_{\sigma_{\eta} \sigma_{\eta}}= & m_{\eta}^{2}+\frac{1}{4}\left(\left(g_{Y X} g_{X}+g_{X}^{2}\right)\left(v_{d}^{2}-v_{u}^{2}\right)+2 g_{X}^{2}\left(v_{\eta}^{2}-v_{\bar{\eta}}^{2}\right)\right)+\frac{1}{2}\left(v_{\bar{\eta}}^{2}+v_{S}^{2}\right)\left|\lambda_{C}\right|^{2}, \\
m_{\sigma_{\eta} \sigma_{\bar{\eta}}}= & \frac{1}{2}\left(\left(-2 l_{W}+\lambda_{H} v_{d} v_{u}\right) \lambda_{C}-\sqrt{2} v_{S}\left(2 M_{S} \lambda_{C}+T_{\lambda_{C}}\right)-v_{S}^{2} \lambda_{C} \kappa\right), \\
m_{\sigma_{\bar{\eta}} \sigma_{\bar{\eta}}}= & m_{\bar{\eta}}^{2}+\frac{1}{4}\left(\left(g_{Y X} g_{X}+g_{X}^{2}\right)\left(v_{u}^{2}-v_{d}^{2}\right)+2 g_{X}^{2}\left(v_{\bar{\eta}}^{2}-v_{\eta}^{2}\right)\right)+\frac{1}{2}\left(v_{\eta}^{2}+v_{S}^{2}\right)\left|\lambda_{C}\right|^{2}, \\
m_{\sigma_{d} \sigma_{s}}= & -v_{u}\left(\left(\kappa v_{S}+\sqrt{2} M_{S}\right) \lambda_{H}-\frac{1}{\sqrt{2}} T_{\lambda_{H}}\right), \quad m_{\sigma_{d} \sigma_{\eta}}=-\frac{1}{2} v_{u} v_{\bar{\eta}} \lambda_{H} \lambda_{C},
\end{aligned}
$$




$$
\begin{array}{rlrl}
m_{\sigma_{u} \sigma_{s}}= & -v_{d}\left(\left(\kappa v_{S}+\sqrt{2} M_{S}\right) \lambda_{H}-\frac{1}{\sqrt{2}} T_{\lambda_{H}}\right), & m_{\sigma_{u} \sigma_{\eta}}=-\frac{1}{2} v_{d} v_{\bar{\eta}} \lambda_{H} \lambda_{C}, \\
m_{\sigma_{\eta} \sigma_{s}}= & v_{\bar{\eta}}\left(\left(\kappa v_{S}+\sqrt{2} M_{S}\right) \lambda_{C}-\frac{1}{\sqrt{2}} T_{\lambda_{C}}\right), & m_{\sigma_{d} \sigma_{\bar{\eta}}}=-\frac{1}{2} v_{u} v_{\eta} \lambda_{H} \lambda_{C}, \\
m_{\sigma_{\bar{\eta}} \sigma_{s}}= & v_{\eta}\left(\left(\kappa v_{S}+\sqrt{2} M_{S}\right) \lambda_{C}-\frac{1}{\sqrt{2}} T_{\lambda_{C}}\right), & m_{\sigma_{u} \sigma_{\bar{\eta}}}=-\frac{1}{2} v_{d} v_{\eta} \lambda_{H} \lambda_{C}, \\
m_{\sigma_{s} \sigma_{s}}= & m_{S}^{2}+4\left|M_{S}\right|^{2}+\left(\kappa v_{S}^{2}-2 l_{W}-\lambda_{C} v_{\eta} v_{\bar{\eta}}+\lambda_{H} v_{d} v_{u}\right) \kappa-2 B_{S} \\
& +\frac{1}{2}\left|\lambda_{C}\right|^{2} \xi^{2}+\frac{1}{2}\left|\lambda_{H}\right|^{2} v^{2}+\sqrt{2} v_{S}\left(2 M_{S} \kappa-T_{\kappa}\right) .
\end{array}
$$

The mass matrix for slepton with the basis $\left(\tilde{e}_{L}, \tilde{e}_{R}\right)$ is diagonalized by $Z^{E}$ through the formula $Z^{E} m_{\tilde{e}}^{2} Z^{E, \dagger}=m_{2, \tilde{e}}^{\text {diag }}$,

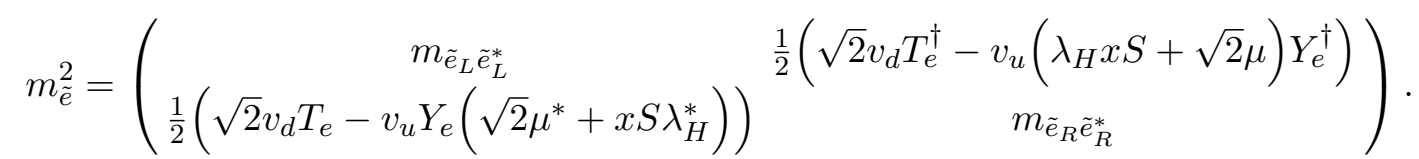

$$
\begin{aligned}
& m_{\tilde{e}_{L} \tilde{e}_{L}^{*}}=m_{\tilde{l}}^{2}+\frac{1}{8}\left(\left(g_{1}^{2}+g_{Y X}^{2}+g_{Y X} g_{X}-g_{2}^{2}\right)\left(v_{d}^{2}-v_{u}^{2}\right)+2 g_{Y X} g_{X}\left(v_{\eta}^{2}-v_{\bar{\eta}}^{2}\right)\right)+\frac{1}{2} v_{d}^{2} Y_{e}^{\dagger} Y_{e} \\
& m_{\tilde{e}_{R} \tilde{e}_{R}^{*}}=m_{e}^{2}-\frac{1}{8}\left(\left[2\left(g_{1}^{2}+g_{Y X}^{2}\right)+3 g_{Y X} g_{X}+g_{X}^{2}\right]\left(v_{d}^{2}-v_{u}^{2}\right)\right. \\
& \left.+\left(4 g_{Y X} g_{X}+2 g_{X}^{2}\right)\left(v_{\eta}^{2}-v_{\bar{\eta}}^{2}\right)\right)+\frac{1}{2} v_{d}^{2} Y_{e} Y_{e}^{\dagger} .
\end{aligned}
$$

The mass matrix for neutralino in the basis $\left(\lambda_{\tilde{B}}, \tilde{W}^{0}, \tilde{H}_{d}^{0}, \tilde{H}_{u}^{0}, \lambda_{\tilde{X}}, \tilde{\eta}, \tilde{\bar{\eta}}, \tilde{s}\right)$ is,

$$
\begin{aligned}
& m_{\tilde{\chi}^{0}}=\left(\begin{array}{cccccccc}
M_{1} & 0 & -\frac{g_{1}}{2} v_{d} & \frac{g_{1}}{2} v_{u} & M_{B B^{\prime}} & 0 & 0 & 0 \\
0 & M_{2} & \frac{1}{2} g_{2} v_{d} & -\frac{1}{2} g_{2} v_{u} & 0 & 0 & 0 & 0 \\
-\frac{g_{1}}{2} v_{d} & \frac{1}{2} g_{2} v_{d} & 0 & m_{\tilde{H}_{u}^{0} \tilde{H}_{d}^{0}} & m_{\lambda_{\tilde{X}} \tilde{H}_{d}^{0}} & 0 & 0 & -\frac{\lambda_{H} v_{u}}{\sqrt{2}} \\
\frac{g_{1}}{2} v_{u} & -\frac{1}{2} g_{2} v_{u} & m_{\tilde{H}_{d}^{0} \tilde{H}_{u}^{0}} & 0 & m_{\lambda_{\tilde{X}} \tilde{H}_{u}^{0}} & 0 & 0 & -\frac{\lambda_{H} v_{d}}{\sqrt{2}} \\
M_{B B^{\prime}} & 0 & m_{\tilde{H}_{d}^{0} \lambda_{\tilde{X}}} & m_{\tilde{H}_{u}^{0} \lambda_{\tilde{X}}} & M_{B L} & -g_{X} v_{\eta} & g_{X} v_{\bar{\eta}} & 0 \\
0 & 0 & 0 & 0 & -g_{X} v_{\eta} & 0 & \frac{1}{\sqrt{2}} \lambda_{C} v_{S} & \frac{1}{\sqrt{2}} \lambda_{C} v_{\bar{\eta}} \\
0 & 0 & 0 & 0 & g_{X} v_{\bar{\eta}} & \frac{1}{\sqrt{2}} \lambda_{C} v_{S} & 0 & \frac{1}{\sqrt{2}} \lambda_{C} v_{\eta} \\
0 & 0 & -\frac{\lambda_{H} v_{u}}{\sqrt{2}} & -\frac{\lambda_{H} v_{d}}{\sqrt{2}} & 0 & \frac{1}{\sqrt{2}} \lambda_{C} v_{\bar{\eta}} & \frac{1}{\sqrt{2}} \lambda_{C} v_{\eta} & m_{\tilde{s} \tilde{s}}
\end{array}\right), \\
& m_{\tilde{H}_{d}^{0} \tilde{H}_{u}^{0}}=-\frac{1}{\sqrt{2}} \lambda_{H} v_{S}-\mu, \quad m_{\tilde{H}_{d}^{0} \lambda_{\tilde{X}}}=-\frac{1}{2}\left(g_{Y X}+g_{X}\right) v_{d}, \\
& m_{\tilde{H}_{u}^{0} \lambda_{\tilde{X}}}=\frac{1}{2}\left(g_{Y X}+g_{X}\right) v_{u}, \quad m_{\tilde{s} \tilde{s}}=2 M_{S}+\sqrt{2} \kappa v_{S} .
\end{aligned}
$$

This matrix is diagonalized by $Z^{N}$

$$
Z^{N *} m_{\tilde{\chi}^{0}} Z^{N \dagger}=m_{\tilde{\chi}^{0}}^{\text {diag }} .
$$


Here, we show the needed couplings in this model. The CP-even Higgs couple with CP-even sneutrinos

$$
\begin{aligned}
& \mathcal{L}_{H \tilde{\nu} R \tilde{\nu}}=H_{i} \tilde{\nu}_{j}^{R} \frac{i}{4}\left\{\sum _ { a , b = 1 } ^ { 3 } \left[-2 \sqrt{2} Z_{k b}^{R *} Z_{j 3+a}^{R *}\left(T_{\nu}\right)_{a b} Z_{i 2}^{H}-2 \lambda_{C} v_{S} Z_{k 3+b}^{R *} Z_{j 3+a}^{R *}\left(Y_{X}\right)_{a b} Z_{i 3}^{H}\right.\right. \\
& \left.-2 \sqrt{2} Z_{k 3+b}^{R *} Z_{j 3+a}^{R *}\left(T_{X}\right)_{a b} Z_{i 4}^{H}-2 \lambda_{C} v_{\eta} Z_{k 3+b}^{R *} Z_{j 3+a}^{R *}\left(Y_{X}\right)_{a b} Z_{i 5}^{H}\right]+[j \leftrightarrow k] \\
& -16 v_{\bar{\eta}} \sum_{a, b, c=1}^{3} Z_{k 3+c}^{R *} Z_{j 3+b}^{R *}\left(Y_{X}\right)_{a c}\left(Y_{X}\right)_{a b} Z_{i 4}^{H}+\sum_{a=1}^{3} Z_{k a}^{R *} Z_{j a}^{R *}\left[\left(g_{Y X} g_{X}+g_{1}^{2}\right.\right. \\
& \left.\left.+g_{Y X}^{2}+g_{2}^{2}\right)\left(-v_{d} Z_{i 1}^{H}+v_{u} Z_{i 2}^{H}\right)-2 g_{Y X} g_{X}\left(-v_{\bar{\eta}} Z_{i 4}^{H}+v_{\eta} Z_{i 3}^{H}\right)\right] \\
& \left.+\sum_{a=1}^{3} Z_{k 3+a}^{R *} Z_{j 3+a}^{R *}\left[\left(g_{Y X} g_{X}+g_{X}^{2}\right)\left(v_{u} Z_{i 2}^{H}-v_{d} Z_{i 1}^{H}\right)-2 g_{X}^{2}\left(v_{\eta} Z_{i 3}^{H}-v_{\bar{\eta}} Z_{i 4}^{H}\right)\right]\right\} \tilde{\nu}_{k}^{* R} .
\end{aligned}
$$

The coupling of two CP-even Higgs and two CP-even sneutrinos reads as

$$
\begin{aligned}
& \mathcal{L}_{H H \tilde{\nu}^{R} \tilde{\nu}^{R}}=H_{i} \tilde{\nu}_{l}^{R}\left\{\frac{i}{2} \sum_{a, b=1}^{3}\left[\left(-\lambda_{C} Z_{l 3+b}^{R *} Z_{k 3+a}^{R *}\left(Y_{X}\right)_{a b}\left(Z_{i 5}^{H} Z_{j 3}^{H}+Z_{i 3}^{H} Z_{j 5}^{H}\right)\right)+(l \leftrightarrow k)\right]\right. \\
& +\frac{i}{4} \sum_{a=1}^{3} Z_{l 3+a}^{R *} Z_{k 3+a}^{R *}\left[\left(g_{Y X} g_{X}+g_{X}^{2}\right)\left(Z_{i 2}^{H} Z_{j 2}^{H}-Z_{i 1}^{H} Z_{j 1}^{H}\right)-2 g_{X}^{2}\left(Z_{i 3}^{H} Z_{j 3}^{H}-Z_{i 4}^{H} Z_{j 4}^{H}\right)\right] \\
& +\frac{i}{4} \sum_{a=1}^{3} Z_{l a}^{R *} Z_{k a}^{R *}\left[\left(g_{Y X} g_{X}+g_{1}^{2}+g_{Y X}^{2}+g_{2}^{2}\right)\left(-Z_{i 1}^{H} Z_{j 1}^{H}+Z_{i 2}^{H} Z_{j 2}^{H}\right)\right. \\
& \left.\left.-2 g_{Y X} g_{X}\left(Z_{i 3}^{H} Z_{j 3}^{H}-Z_{i 4}^{H} Z_{j 4}^{H}\right)\right]-4 i \sum_{a, b, c=1}^{3} Z_{l 3+c}^{R *} Z_{k 3+b}^{R *}\left(Y_{X}\right)_{a b}\left(Y_{X}\right)_{a c} Z_{i 4}^{H} Z_{j 4}^{H}\right\} H_{k} \tilde{\nu}_{k}^{R} .
\end{aligned}
$$

The other used vertexes including the couplings of: $H-H-H, H-W-W$ and $H-$ $Z-Z$ are

$$
\begin{aligned}
& \mathcal{L}_{H H H}=i H_{i} H_{j}\left\{\left(\frac{1}{4} g_{1}^{2}+\frac{1}{4} g_{Y X}^{2}+\frac{1}{4} g_{2}^{2}+\frac{1}{2} g_{Y X} g_{X}+\frac{1}{4} g_{X}^{2}-\lambda_{H}^{2}\right)\left[v_{u}\langle 112\rangle+v_{d}\langle 122\rangle\right]\right. \\
& -\left(\frac{3}{4} g_{1}^{2}+\frac{3}{4} g_{Y X}^{2}+\frac{3}{4} g_{2}^{2}+\frac{3}{2} g_{Y X} g_{X}+\frac{3}{4} g_{X}^{2}\right)\left[v_{u}\langle 111\rangle+v_{d}\langle 222\rangle\right]+\frac{1}{2}\left(g_{Y X} g_{X}+g_{X}^{2}\right) \\
& \times\left[v_{\bar{\eta}}(\langle 114\rangle+\langle 224\rangle)-v_{\eta}(\langle 113\rangle+\langle 223\rangle)+v_{u}(\langle 233\rangle+\langle 244\rangle)-v_{d}(\langle 133\rangle+\langle 144\rangle)\right] \\
& -\left(v_{S} \lambda_{H}^{2}+\sqrt{2} \mu \lambda_{H}\right)(\langle 115\rangle+\langle 225\rangle)+\left(\lambda_{H} v_{S} \kappa+\sqrt{2} M_{S} \lambda_{H}+\frac{1}{\sqrt{2}} T_{\lambda_{H}}\right)\langle 125\rangle
\end{aligned}
$$




$$
\begin{aligned}
& -\left(\lambda_{C} v_{S} \kappa+\sqrt{2} M_{S} \lambda_{C}+\frac{1}{\sqrt{2}} T_{\lambda_{C}}\right)\langle 345\rangle+\frac{1}{2} \lambda_{H} \lambda_{C}\left[v_{\bar{\eta}}\langle 123\rangle+v_{\eta}\langle 124\rangle+v_{u}\langle 134\rangle\right. \\
& \left.+v_{d}\langle 234\rangle\right]+\left(\lambda_{H} v_{u} \kappa-v_{d} \lambda_{H}^{2}\right)\langle 155\rangle+\left(\lambda_{H} v_{d} \kappa-v_{u} \lambda_{H}^{2}\right)\langle 255\rangle-3 g_{X}^{2}\left(v_{\eta}\langle 333\rangle+v_{\bar{\eta}}\langle 444\rangle\right) \\
& +\left(g_{X}^{2}-\lambda_{C}^{2}\right)\left(v_{\eta}\langle 344\rangle+v_{\bar{\eta}}\langle 334\rangle\right)-v_{S} \lambda_{C}^{2}(\langle 335\rangle+\langle 445\rangle)-\left(\lambda_{C}^{2} v_{\eta}+\lambda_{C} v_{\bar{\eta}} \kappa\right)\langle 355\rangle \\
& \left.-\left(\lambda_{C}^{2} v_{\bar{\eta}}+\lambda_{C} v_{\eta} \kappa\right)\langle 455\rangle-\left(6 v_{S} \kappa^{2}+6 \sqrt{2} M_{S} \kappa+\sqrt{2} T_{\kappa}\right)\langle 555\rangle\right\} H_{k} \\
& \mathcal{L}_{H W W}=H_{i} W_{\mu}\left(\frac{i}{2} g_{2}^{2}\left(v_{d} Z_{i 1}^{H}+v_{u} Z_{i 2}^{H}\right) g^{\sigma \mu}\right) W_{\sigma}^{*}, \\
& \mathcal{L}_{H Z Z}=H_{i} Z_{\mu}\left\{\frac { i } { 2 } \left[\left(g_{1} \cos \theta_{W}^{\prime} \sin \theta_{W}+g_{2} \cos \theta_{W}^{\prime} \cos \theta_{W}-g_{Y X} g_{X} \sin \theta_{W}^{\prime}\right)^{2}\right.\right. \\
& \left.\left.\times\left(v_{d} Z_{i 1}^{H}+v_{u} Z_{i 2}^{H}\right)+4\left(g_{X} \sin \theta_{W}^{\prime}\right)^{2}\left(v_{\bar{\eta}} Z_{i 4}^{H}+v_{\eta} Z_{i 3}^{H}\right)\right] g^{\sigma \mu}\right\} Z_{\sigma}^{*} .
\end{aligned}
$$

Here $\langle\alpha \alpha \alpha\rangle,\langle\alpha \alpha \beta\rangle,\langle\alpha \beta \gamma\rangle$ are the shorthand notations

$$
\begin{aligned}
\langle\alpha \alpha \alpha\rangle= & Z_{i \alpha}^{H} Z_{j \alpha}^{H} Z_{k \alpha}^{H}, \quad\langle\alpha \alpha \beta\rangle=Z_{i \alpha}^{H} Z_{j \alpha}^{H} Z_{k \beta}^{H}+Z_{i \alpha}^{H} Z_{j \beta}^{H} Z_{k \alpha}^{H}+Z_{i \beta}^{H} Z_{j \alpha}^{H} Z_{k \alpha}^{H}, \quad(\alpha \neq \beta), \\
\langle\alpha \beta \gamma\rangle= & Z_{i \alpha}^{H} Z_{j \gamma}^{H} Z_{k \beta}^{H}+Z_{i \gamma}^{H} Z_{j \alpha}^{H} Z_{k \beta}^{H}+Z_{i \alpha}^{H} Z_{j \beta}^{H} Z_{k \gamma}^{H}+Z_{i \gamma}^{H} Z_{j \beta}^{H} Z_{k \alpha}^{H}+Z_{i \beta}^{H} Z_{j \alpha}^{H} Z_{k \gamma}^{H} \\
& +Z_{i \beta}^{H} Z_{j \gamma}^{H} Z_{k \alpha}^{H}, \quad(\alpha \neq \beta \neq \gamma) .
\end{aligned}
$$

Some other used couplings are shown as

$$
\begin{aligned}
& \mathcal{L}_{W W \tilde{\nu}^{R} \tilde{\nu}^{R}}=\tilde{\nu}_{i}^{R} W_{\nu}\left(\frac{i}{2} g_{2}^{2} \sum_{a=1}^{3} Z_{i a}^{R *} Z_{j a}^{R *} g^{\mu \nu}\right) \tilde{\nu}_{j}^{R} W_{\mu} \\
& \mathcal{L}_{Z Z \tilde{\nu}^{R} \tilde{\nu}^{R}}=\tilde{\nu}_{i}^{R} Z_{\nu}\left\{i \sum _ { a = 1 } ^ { 3 } \left[Z _ { i a } ^ { R * } Z _ { j a } ^ { R * } \left(\frac{1}{2} g_{2}^{2}\left(\cos \theta_{W} \cos \theta_{W}^{\prime}\right)^{2}+\frac{1}{2} g_{1}^{2}\left(\sin \theta_{W} \cos \theta_{W}^{\prime}\right)^{2}\right.\right.\right. \\
& +g_{1} g_{2} \cos \theta_{W} \sin \theta_{W}\left(\cos \theta_{W}^{\prime}\right)^{2}-g_{Y X} \sin \theta_{W}^{\prime} \cos \theta_{W}^{\prime}\left(g_{2} \cos \theta_{W}+g_{1} \sin \theta_{W}\right) \\
& \left.\left.\left.+\frac{1}{2} g_{Y X}^{2}\left(\sin \theta_{W}^{\prime}\right)^{2}\right)+\frac{1}{2} g_{X}^{2}\left(\sin \theta_{W}^{\prime}\right)^{2} Z_{i 3+a}^{R *} Z_{j 3+a}^{R *}\right] g^{\mu \nu}\right\} \tilde{\nu}_{j}^{R} Z_{\mu} \\
& \mathcal{L}_{\tilde{e} \tilde{\nu} R * W}=\tilde{e}_{i} \tilde{\nu}_{j}^{R *}\left(-\frac{i}{2} g_{2} \sum_{a=1}^{3} Z_{i a}^{E *} Z_{j a}^{R *}\left(-p_{\mu}^{\tilde{\nu}_{j}^{R}}+p_{\mu}^{\tilde{e}_{i}}\right)\right) W^{\mu}+h . c \\
& \mathcal{L}_{Z d d}=\bar{d}\left[\frac{i}{6}\left(3 g_{2} \cos \theta_{W} \cos \theta_{W}^{\prime}+g_{1} \sin \theta_{W} \cos \theta_{W}^{\prime}-g_{Y X} \sin \theta_{W}^{\prime}\right) \gamma_{\mu} P_{L}\right. \\
& \left.-\frac{i}{6}\left(2 g_{1} \sin \theta_{W} \cos \theta_{W}^{\prime}-\left(2 g_{Y X}+3 g_{X}\right) \sin \theta_{W}^{\prime}\right) \gamma_{\mu} P_{R}\right] d Z^{\mu}
\end{aligned}
$$


$\mathcal{L}_{Z^{\prime} d d}=\bar{d}\left[-\frac{i}{6}\left(3 g_{2} \cos \theta_{W} \sin \theta_{W}^{\prime}+g_{1} \sin \theta_{W} \sin \theta_{W}^{\prime}+g_{Y X} \cos \theta_{W}^{\prime}\right) \gamma_{\mu} P_{L}\right.$

$\left.+\frac{i}{6}\left[2 g_{1} \sin \theta_{W} \sin \theta_{W}^{\prime}+\left(2 g_{Y X}+3 g_{X}\right) \cos \theta_{W}^{\prime}\right] \gamma_{\mu} P_{R}\right] d Z^{\prime \mu}$,

$\mathcal{L}_{Z l l}=\bar{l}\left\{\frac{i}{2}\left(-g_{1} \sin \theta_{W} \cos \theta_{W}^{\prime}+g_{2} \cos \theta_{W} \cos \theta_{W}^{\prime}+g_{Y X} \sin \theta_{W}^{\prime}\right) \gamma_{\mu} P_{L}\right.$

$\left.-\frac{i}{2}\left(2 g_{1} \sin \theta_{W} \cos \theta_{W}^{\prime}-\left(2 g_{Y X}+g_{X}\right) \sin \theta_{W}^{\prime}\right) \gamma_{\mu} P_{R}\right\} l Z^{\mu}$,

$\mathcal{L}_{Z^{\prime} l l}=\bar{l}\left\{\frac{i}{2}\left(g_{1} \sin \theta_{W} \sin \theta_{W}^{\prime}-g_{2} \cos \theta_{W} \sin \theta_{W}^{\prime}+g_{Y X} \cos \theta_{W}^{\prime}\right) \gamma_{\mu} P_{L}\right.$

$\left.+\frac{i}{2}\left(2 g_{1} \sin \theta_{W} \sin \theta_{W}^{\prime}+\left(2 g_{Y X}+g_{X}\right) \cos \theta_{W}^{\prime}\right) \gamma_{\mu} P_{R}\right\} l Z^{\prime \mu}$

$\mathcal{L}_{Z u u}=\bar{u}\left\{-\frac{i}{6}\left(3 g_{2} \cos \theta_{W} \cos \theta_{W}^{\prime}-g_{1} \sin \theta_{W} \cos \theta_{W}^{\prime}+g_{Y X} \sin \theta_{W}^{\prime}\right) \gamma_{\mu} P_{L}\right.$

$\left.+\frac{i}{6}\left[-\left(4 g_{Y X}+3 g_{X}\right) \sin \theta_{W}^{\prime}+4 g_{1} \sin \theta_{W} \cos \theta_{W}^{\prime}\right] \gamma_{\mu} P_{R}\right\} u Z^{\mu}$,

$\mathcal{L}_{Z^{\prime} u u}=\bar{u}\left\{-\frac{i}{6}\left(-3 g_{2} \cos \theta_{W} \sin \theta_{W}^{\prime}+g_{1} \sin \theta_{W} \sin \theta_{W}^{\prime}+g_{Y X} \cos \theta_{W}^{\prime}\right) \gamma_{\mu} P_{L}\right.$

$\left.-\frac{i}{6}\left[\left(4 g_{Y X}+3 g_{X}\right) \cos \theta_{W}^{\prime}+4 g_{1} \sin \theta_{W} \sin \theta_{W}^{\prime}\right] \gamma_{\mu} P_{R}\right\} u Z^{\prime \mu}$,

$\mathcal{L}_{Z \nu \nu}=\bar{\nu}_{i}\left\{-\frac{i}{2}\left(g_{1} \sin \theta_{W} \cos \theta_{W}^{\prime}+g_{2} \cos \theta_{W} \cos \theta_{W}^{\prime}-g_{Y X} \sin \theta_{W}^{\prime}\right) \sum_{a=1}^{3} U_{j a}^{V *} U_{i a}^{V} \gamma_{\mu} P_{L}\right.$

$\left.+\frac{i}{2}\left(g_{1} \sin \theta_{W} \cos \theta_{W}^{\prime}+g_{2} \cos \theta_{W} \cos \theta_{W}^{\prime}-g_{Y X} \sin \theta_{W}^{\prime}\right) \sum_{a=1}^{3} U_{j a}^{V} U_{i a}^{V *} \gamma_{\mu} P_{R}\right\} \nu_{j} Z^{\mu}$,

$\mathcal{L}_{Z^{\prime} \nu \nu}=\bar{\nu}_{i}\left\{\frac{i}{2}\left(g_{1} \sin \theta_{W} \sin \theta_{W}^{\prime}+g_{2} \cos \theta_{W} \sin \theta_{W}^{\prime}+g_{Y X} \cos \theta_{W}^{\prime}\right) \sum_{a=1}^{3} U_{j a}^{V *} U_{i a}^{V} \gamma_{\mu} P_{L}\right.$

$\left.-\frac{i}{2}\left(g_{1} \sin \theta_{W} \sin \theta_{W}^{\prime}+g_{2} \cos \theta_{W} \sin \theta_{W}^{\prime}+g_{Y X} \cos \theta_{W}^{\prime}\right) \sum_{a=1}^{3} U_{i a}^{V *} U_{j a}^{V} \gamma_{\mu} P_{R}\right\} \nu_{j} Z^{\prime \mu}$,

$\mathcal{L}_{\tilde{\nu}^{I} \tilde{\nu}^{R} Z}=\tilde{\nu}_{i}^{I} \tilde{\nu}_{j}^{R}\left\{\frac{1}{2}\left(-p_{\mu}^{\tilde{\nu}_{j}^{R}}+p_{\mu}^{\tilde{\nu}_{i}^{I}}\right)\left[\left(g_{1} \sin \theta_{W} \cos \theta_{W}^{\prime}+g_{2} \cos \theta_{W} \cos \theta_{W}^{\prime}\right.\right.\right.$

$\left.\left.\left.-g_{Y X} \sin \theta_{W}^{\prime}\right) \sum_{a=1}^{3} Z_{i a}^{I *} Z_{j a}^{R *}+g_{X} \sin \theta_{W}^{\prime} \sum_{a=1}^{3} Z_{i 3+a}^{I *} Z_{j 3+a}^{R *}\right]\right\} Z^{\mu}$ 


$$
\begin{aligned}
& \mathcal{L}_{\tilde{\nu}^{I} \tilde{\nu}^{R} Z}=\tilde{\nu}_{i}^{I} \tilde{\nu}_{j}^{R}\left\{\frac { 1 } { 2 } ( - p _ { \mu } ^ { \tilde { \nu } _ { j } ^ { R } } + p _ { \mu } ^ { \tilde { \nu } _ { i } ^ { I } } ) \left[-\left(g_{1} \sin \theta_{W} \sin \theta_{W}^{\prime}+g_{2} \cos \theta_{W} \sin \theta_{W}^{\prime}\right.\right.\right. \\
& \left.\left.\left.+g_{Y X} \cos \theta_{W}^{\prime}\right) \sum_{a=1}^{3} Z_{i a}^{I *} Z_{j a}^{R *}+g_{X} \cos \theta_{W}^{\prime} \sum_{a=1}^{3} Z_{i 3+a}^{I *} Z_{j 3+a}^{R *}\right]\right\} Z^{\prime \mu} .
\end{aligned}
$$

Open Access. This article is distributed under the terms of the Creative Commons Attribution License (CC-BY 4.0), which permits any use, distribution and reproduction in any medium, provided the original author(s) and source are credited.

\section{References}

[1] Planck collaboration, Planck 2013 results. XVI. Cosmological parameters, Astron. Astrophys. 571 (2014) A16 [arXiv:1303.5076] [INSPIRE].

[2] R.H. Cyburt, Primordial nucleosynthesis for the new cosmology: Determining uncertainties and examining concordance, Phys. Rev. D 70 (2004) 023505 [astro-ph/0401091] [INSPIRE].

[3] G. Bertone, D. Hooper and J. Silk, Particle dark matter: Evidence, candidates and constraints, Phys. Rept. 405 (2005) 279 [hep-ph/0404175] [inSPIRE].

[4] E. Corbelli and P. Salucci, The Extended Rotation Curve and the Dark Matter Halo of M33, Mon. Not. Roy. Astron. Soc. 311 (2000) 441 [astro-ph/9909252] [INSPIRE].

[5] D. Clowe et al., A direct empirical proof of the existence of dark matter, Astrophys. J. 648 (2006) L109 [astro-ph/0608407] [INSPIRE].

[6] A. Taylor, S. Dye, T.J. Broadhurst, N. Benitez and E. van Kampen, Gravitational lens magnification and the mass of abell 1689, Astrophys. J. 501 (1998) 539 [astro-ph/9801158] [INSPIRE].

[7] D. Walsh, R.F. Carswell, R.J. Weymann, 0957 + 561 A, B: twin quasistellar objects or gravitational lens?, Nature 279 (1979) 381 [INSPIRE].

[8] J.L. Feng, Dark Matter Candidates from Particle Physics and Methods of Detection, Ann. Rev. Astron. Astrophys. 48 (2010) 495 [arXiv: 1003. 0904] [INSPIRE].

[9] M. Drees and M.M. Nojiri, The Neutralino relic density in minimal $N=1$ supergravity, Phys. Rev. D 47 (1993) 376 [hep-ph/9207234] [INSPIRE].

[10] L.-B. Jia, Dark photon portal dark matter with the $21 \mathrm{~cm}$ anomaly, Eur. Phys. J. C 79 (2019) 80 [arXiv:1804.07934] [INSPIRE].

[11] CMS collaboration, Observation of a New Boson at a Mass of $125 \mathrm{GeV}$ with the CMS Experiment at the LHC, Phys. Lett. B $\mathbf{7 1 6}$ (2012) 30 [arXiv:1207.7235] [INSPIRE].

[12] ATLAS collaboration, Observation of a new particle in the search for the Standard Model Higgs boson with the ATLAS detector at the LHC, Phys. Lett. B 716 (2012) 1 [arXiv: 1207.7214] [INSPIRE].

[13] Particle Data Group, Review of Particle Physics, Phys. Rev. D 98 (2018) 030001 [INSPIRE].

[14] S. Andreas, T. Hambye and M.H.G. Tytgat, WIMP dark matter, Higgs exchange and DAMA, JCAP 10 (2008) 034 [arXiv:0808.0255] [INSPIRE].

[15] J.-J. Cao, Z.X. Heng, J.M. Yang and J. Zhu, Higgs decay to dark matter in low energy SUSY: is it detectable at the LHC?, JHEP 06 (2012) 145 [arXiv: 1203.0694] [INSPIRE]. 
[16] J. Rosiek, Complete set of Feynman rules for the MSSM - ERRATUM, hep-ph/9511250 [Original paper: Phys. Rev. D 41 (1990) 3464, inSPIRE].

[17] Z. Thomas, D. Tucker-Smith and N. Weiner, Mixed Sneutrinos, Dark Matter and the CERN LHC, Phys. Rev. D 77 (2008) 115015 [arXiv:0712.4146] [INSPIRE].

[18] T2K collaboration, Indication of Electron Neutrino Appearance from an Accelerator-produced Off-axis Muon Neutrino Beam, Phys. Rev. Lett. 107 (2011) 041801 [arXiv: 1106.2822] [INSPIRE].

[19] MINOS collaboration, Improved search for muon-neutrino to electron-neutrino oscillations in MINOS, Phys. Rev. Lett. 107 (2011) 181802 [arXiv:1108.0015] [INSPIRE].

[20] A. Ghosh, T. Mondal and B. Mukhopadhyaya, Right sneutrino with $\Delta L=2$ masses as nonthermal dark matter, Phys. Rev. D 99 (2019) 035018 [arXiv:1807.04964] [INSPIRE].

[21] C. Arina and N. Fornengo, Sneutrino cold dark matter, a new analysis: Relic abundance and detection rates, JHEP 11 (2007) 029 [arXiv:0709.4477] [INSPIRE].

[22] C. Arina, F. Bazzocchi, N. Fornengo, J.C. Romao and J.W.F. Valle, Minimal supergravity sneutrino dark matter and inverse seesaw neutrino masses, Phys. Rev. Lett. 101 (2008) 161802 [arXiv: 0806 .3225] [INSPIRE].

[23] H.N. Long, Right-handed sneutrinos as self-interacting dark matter in supersymmetric economical 3-3-1 model, Adv. Stud. Theor. Phys. 4 (2010) 173 [arXiv:0710.5833] [InSPIRE].

[24] D.G. Cerdeno and O. Seto, Right-handed sneutrino dark matter in the NMSSM, JCAP 08 (2009) 032 [arXiv:0903.4677] [INSPIRE].

[25] J.-J. Cao, X.F. Guo, Y.L. He, L. Shang and Y. Yue, Sneutrino DM in the NMSSM with inverse seesaw mechanism, JHEP 10 (2017) 044 [arXiv:1707.09626] [INSPIRE].

[26] J.-J. Cao, J. Li, Y.S. Pan, L. Shang, Y. Yue and D. Zhang, Bayesian analysis of sneutrino dark matter in the NMSSM with a type-I seesaw mechanism, Phys. Rev. D 99 (2019) 115033 [arXiv: 1807.03762] [INSPIRE].

[27] T. Han, H.K. Liu, S. Mukhopadhyay and X. Wang, Dark Matter Blind Spots at One-Loop, JHEP 03 (2019) 080 [arXiv: 1810.04679] [INSPIRE].

[28] J. March-Russell, C. McCabe and M. McCullough, Neutrino-Flavoured Sneutrino Dark Matter, JHEP 03 (2010) 108 [arXiv:0911.4489] [INSPIRE].

[29] D.A. Demir, L.L. Everett, M. Frank, L. Selbuz and I. Turan, Sneutrino Dark Matter: Symmetry Protection and Cosmic Ray Anomalies, Phys. Rev. D 81 (2010) 035019 [arXiv:0906.3540] [INSPIRE].

[30] Z.F. Kang, J. Li, T. Li, T.-J. Liu and J.M. Yang, The maximal U(1) $)_{L}$ inverse seesaw from $d=5$ operator and oscillating asymmetric Sneutrino dark matter, Eur. Phys. J. C 76 (2016) 270 [arXiv: 1102.5644] [INSPIRE].

[31] D.G. Cerdeno, J.-H. Huh, M. Peiro and O. Seto, Very light right-handed sneutrino dark matter in the NMSSM, JCAP 11 (2011) 027 [arXiv:1108.0978] [INSPIRE].

[32] B. Zhu, R. Ding and Y. Li, Realization of Sneutrino Self-interacting Dark Matter in the Focus Point Supersymmetry, Phys. Rev. D 98 (2018) 035007 [arXiv: 1804.00277] [INSPIRE].

[33] J. Chang, K.M. Cheung, H. Ishida, C.-T. Lu, M. Spinrath and Y.-L.S. Tsai, Sneutrino Dark Matter via pseudoscalar X-funnel meets Inverse Seesaw, JHEP 09 (2018) 071 [arXiv: 1806.04468] [INSPIRE]. 
[34] D.K. Ghosh, K. Huitu, S. Mondal and M. Mitra, Same-sign trilepton signal for stop quark in the presence of sneutrino dark matter, Phys. Rev. D 99 (2019) 075014 [arXiv:1807.07385] [INSPIRE].

[35] H.-S. Lee, K.T. Matchev and S. Nasri, Revival of the thermal sneutrino dark matter, Phys. Rev. D 76 (2007) 041302 [hep-ph/0702223] [InSPIRE].

[36] P. Bandyopadhyay, E.J. Chun and J.-C. Park, Right-handed sneutrino dark matter in U(1)' seesaw models and its signatures at the LHC, JHEP 06 (2011) 129 [arXiv:1105.1652] [INSPIRE].

[37] G. Bélanger, J. Da Silva and A. Pukhov, The Right-handed sneutrino as thermal dark matter in U(1) extensions of the MSSM, JCAP 12 (2011) 014 [arXiv:1110.2414] [INSPIRE].

[38] G. Bélanger, J. Da Silva, U. Laa and A. Pukhov, Probing U(1) extensions of the MSSM at the LHC Run I and in dark matter searches, JHEP 09 (2015) 151 [arXiv:1505. 06243] [INSPIRE].

[39] G. Bélanger, J. Da Silva and H.M. Tran, Dark matter in U(1) extensions of the MSSM with gauge kinetic mixing, Phys. Rev. D 95 (2017) 115017 [arXiv:1703.03275] [INSPIRE].

[40] F. Staub, SARAH, arXiv:0806.0538 [INSPIRE].

[41] F. Staub, SARAH 4: A tool for (not only SUSY) model builders, Comput. Phys. Commun. 185 (2014) 1773 [arXiv: 1309.7223] [INSPIRE].

[42] F. Staub, Exploring new models in all detail with SARAH, Adv. High Energy Phys. 2015 (2015) 840780 [arXiv: 1503.04200] [INSPIRE].

[43] H.E.S.S. collaboration, The energy spectrum of cosmic-ray electrons at TeV energies, Phys. Rev. Lett. 101 (2008) 261104 [arXiv:0811.3894] [INSPIRE].

[44] O. Adriani et al., A new measurement of the antiproton-to-proton flux ratio up to $100 \mathrm{GeV}$ in the cosmic radiation, Phys. Rev. Lett. 102 (2009) 051101 [arXiv:0810.4994] [INSPIRE].

[45] M. Carena, J.R. Espinosa, M. Quirós and C.E.M. Wagner, Analytical expressions for radiatively corrected Higgs masses and couplings in the MSSM, Phys. Lett. B 355 (1995) 209 [hep-ph/9504316] [INSPIRE].

[46] M. Carena, S. Gori, N.R. Shah and C.E.M. Wagner, A 125 GeV SM-like Higgs in the MSSM and the $\gamma \gamma$ rate, JHEP 03 (2012) 014 [arXiv:1112.3336] [INSPIRE].

[47] M.E. Peskin and D.V. Schroeder, An introduction to quantum field theory, Addison Wesley, Reading U.S.A. (1995).

[48] V. Barger, P. Fileviez Pérez and S. Spinner, Minimal gauged U(1) $B-L$ model with spontaneous R parity violation, Phys. Rev. Lett. 102 (2009) 181802 [arXiv:0812.3661] [INSPIRE].

[49] P.H. Chankowski, S. Pokorski and J. Wagner, $Z^{\prime}$ and the Appelquist-Carrazzone decoupling, Eur. Phys. J. C 47 (2006) 187 [hep-ph/0601097] [INSPIRE].

[50] J.-L. Yang, T.-F. Feng, S.-M. Zhao, R.-F. Zhu, X.-Y. Yang and H.-B. Zhang, Two loop electroweak corrections to $\bar{B} \rightarrow X_{s} \gamma$ and $B_{s}^{0} \rightarrow \mu^{+} \mu^{-}$in the B-LSSM, Eur. Phys. J. C 78 (2018) 714 [arXiv: 1803.09904] [INSPIRE].

[51] J. McDonald, Gauge singlet scalars as cold dark matter, Phys. Rev. D 50 (1994) 3637 [hep-ph/0702143] [INSPIRE].

[52] G. Bélanger, F. Boudjema, A. Pukhov and A. Semenov, MicrOMEGAs4.1: two dark matter candidates, Comput. Phys. Commun. 192 (2015) 322 [arXiv:1407.6129] [INSPIRE]. 
[53] G. Jungman, M. Kamionkowski and K. Griest, Supersymmetric dark matter, Phys. Rept. 267 (1996) 195 [hep-ph/9506380] [INSPIRE].

[54] S. Gopalakrishna, A. de Gouvêa and W. Porod, Right-handed sneutrinos as nonthermal dark matter, JCAP 05 (2006) 005 [hep-ph/0602027] [INSPIRE].

[55] X.-G. He, T. Li, X.-Q. Li, J. Tandean and H.-C. Tsai, Constraints on Scalar Dark Matter from Direct Experimental Searches, Phys. Rev. D 79 (2009) 023521 [arXiv:0811.0658] [INSPIRE].

[56] W. Chao, H.-k. Guo and Y. Zhang, Majorana Dark matter with B $+L$ gauge symmetry, JHEP 04 (2017) 034 [arXiv: 1604.01771] [INSPIRE].

[57] S.-M. Zhao, T.-F. Feng, G.-Z. Ning, J.-B. Chen, H.-B. Zhang and X.X. Dong, The extended BLMSSM with a 125 GeV Higgs boson and dark matter, Eur. Phys. J. C 78 (2018) 324 [arXiv: 1711.10731] [INSPIRE].

[58] M. Freytsis and Z. Ligeti, On dark matter models with uniquely spin-dependent detection possibilities, Phys. Rev. D 83 (2011) 115009 [arXiv: 1012.5317] [INSPIRE].

[59] T. Bringmann, J. Edsjö, P. Gondolo, P. Ullio and L. Bergström, DarkSUSY 6: An Advanced Tool to Compute Dark Matter Properties Numerically, JCAP 07 (2018) 033 [arXiv: 1802.03399] [INSPIRE].

[60] G. Bélanger, F. Boudjema, A. Goudelis, A. Pukhov and B. Zaldivar, MicrOMEGAs5.0: Freeze-in, Comput. Phys. Commun. 231 (2018) 173 [arXiv:1801.03509] [INSPIRE].

[61] W. Chao, Direct detections of Majorana dark matter in vector portal, JHEP 11 (2019) 013 [arXiv: 1904.09785] [INSPIRE].

[62] V.D. Barger, K.M. Cheung, K. Hagiwara and D. Zeppenfeld, Global study of electron quark contact interactions, Phys. Rev. D 57 (1998) 391 [hep-ph/9707412] [INSPIRE].

[63] J. Erler and M.J.R. Musolf, Low energy tests of the weak interaction, Prog. Part. Nucl. Phys. 54 (2005) 351 [hep-ph/0404291] [INSPIRE].

[64] CMS collaboration, Search for heavy resonances decaying to tau lepton pairs in proton-proton collisions at $\sqrt{s}=13 \mathrm{TeV}, \mathrm{JHEP} 02$ (2017) 048 [arXiv:1611.06594] [INSPIRE].

[65] D. Pappadopulo, A. Thamm, R. Torre and A. Wulzer, Heavy Vector Triplets: Bridging Theory and Data, JHEP 09 (2014) 060 [arXiv:1402.4431] [INSPIRE].

[66] ATLAS collaboration, Search for new high-mass phenomena in the dilepton final state using $36 \mathrm{fb}^{-1}$ of proton-proton collision data at $\sqrt{s}=13 \mathrm{TeV}$ with the ATLAS detector, JHEP 10 (2017) 182 [arXiv: 1707. 02424] [INSPIRE].

[67] G. Cacciapaglia, C. Csáki, G. Marandella and A. Strumia, The Minimal Set of Electroweak Precision Parameters, Phys. Rev. D 74 (2006) 033011 [hep-ph/0604111] [InSPIRE].

[68] M. Carena, A. Daleo, B.A. Dobrescu and T.M.P. Tait, $Z^{\prime}$ gauge bosons at the Tevatron, Phys. Rev. D 70 (2004) 093009 [hep-ph/0408098] [INSPIRE].

[69] L. Basso, The Higgs sector of the minimal SUSY B - L model, Adv. High Energy Phys. 2015 (2015) 980687 [arXiv: 1504.05328] [INSPIRE].

[70] PandaX-II collaboration, Dark Matter Results From 54-Ton-Day Exposure of PandaX-II Experiment, Phys. Rev. Lett. 119 (2017) 181302 [arXiv:1708.06917] [INSPIRE].

[71] XENON collaboration, First Dark Matter Search Results from the XENON1T Experiment, Phys. Rev. Lett. 119 (2017) 181301 [arXiv:1705.06655] [INSPIRE]. 